\title{
LncRNA-SARCC suppresses renal cell carcinoma (RCC) progression via altering the androgen receptor (AR)/miRNA-143-3p signals
}

\author{
Wei Zhai ${ }^{1,2,3,7}$, Yin Sun ${ }^{3,7}$, Changcheng Guo ${ }^{1,3,7}$, Guanghui Hu${ }^{1}$, Mingchao Wang ${ }^{3,4}$, Jiayi Zheng ${ }^{5}$, WanYing Lin ${ }^{3}$, Qingbo Huang ${ }^{3}$, \\ Gonghui Li ${ }^{4}$, Junhua Zheng ${ }^{*, 1}$ and Chawnshang Chang ${ }^{\star, 3,6}$
}

\begin{abstract}
While the androgen receptor (AR) might promote renal cell carcinoma (RCC) initiation and progression, the molecular mechanisms involved remain largely unclear. Here, we discovered the novel LncRNA-SARCC, which was suppressed and associated with better prognosis in RCC. Preclinical studies using multiple RCC cells and in vivo mouse model indicated that LnCRNA-SARCC could attenuate RCC cell invasion, migration and proliferation in vitro and in vivo. Mechanistically, LnCRNA-SARCC bound and destabilized AR protein with an inhibition of AR function, which led to transcriptionally de-repress miR-143-3p expression, thus inhibition of its downstream signals including AKT, MMP-13, K-RAS and P-ERK. In addition, bisulfite sequencing analysis substantiated that LncRNA-SARCC promoter was highly methylated in renal cancer tissues compared with paired non-cancerous renal tissues. Notably, treating with Sunitinib, the multi-targeted receptor tyrosine kinase inhibitor, increased the expression of LnCRNA-SARCC, which decreased RCC cells resistance to Sunitinib. Thus, our study presented a road map for targeting this newly identified LncRNA-SARCC and its pathway, which expands potential therapeutic strategies for RCC treatment.
\end{abstract}

Cell Death and Differentiation (2017) 24, 1502-1517; doi:10.1038/cdd.2017.74; published online 23 June 2017

Renal cell carcinoma $(\mathrm{RCC})$ is one of the most aggressive human neoplasms in the world, accounting for $\sim 2-3 \%$ of adult malignancies. ${ }^{1,2}$ Surgery remains the unique effective treatment for RCC because of its resistance to radiation-, chemo- and immuno-therapy. ${ }^{3}$ Unfortunately, $\sim 30 \%$ of patients developed metastatic RCC (mRCC) after surgical resection. The vast majority of patients with $\mathrm{mRCC}$ have only a median of 13 months survival time. ${ }^{4}$ The underlying molecular mechanisms that regulate RCC progression are yet to be elucidated. Sunitinib, as the first-line targeted therapy, is a protein tyrosine kinase inhibitor and has been shown to provide clinical benefits in patients with $\mathrm{mRCC}$, however, its effect is still limited for patients with selective pathological types. ${ }^{5}$ Therefore, searching for the better therapy for RCC is urgently needed.

The androgen receptor (AR) was reported to act as an oncogene, promoting RCC progression through the HIF-2a/ VEGF pathway, consistent with the predominance of RCC incidence in males (1.6:1.0 male to female ratio). ${ }^{6-8}$ Long noncoding RNAs (LncRNAs) are non-protein-coding RNA species longer than 200 nucleotides. ${ }^{9,10}$ Recent studies of the human transcriptome attest that an astounding number of LncRNAs might have key roles for the transcriptional regulation linked to cancer progression and are implicated as oncogenes or tumor suppressors. ${ }^{11-14}$ LncRNAs can function as signals, decoys, guides and scaffolding in regulating nuclear activities. ${ }^{15}$ Clinically, LncRNAs can serve as diagnostic or prognostic markers in cancers. ${ }^{16-18}$

Here, we identified LncRNA-ENST00000460407 and concluded that it functioned as a tumor suppressor as well as a potential diagnostic or prognostic marker in RCC. This LncRNA could directly bind to AR and post-transcriptionally suppress AR protein and transactivation to regulate signals such as miR-143-3p/AKT/MMP-13/K-RAS/P-ERK to influence RCC cell invasion, migration and proliferation.

Results

LncRNA-SARCC associated with AR and attenuated AR expression and transactivation. Early studies showed that AR functioned as an oncogene in RCC. ${ }^{6,19}$ We first detected AR protein levels in commonly used human RCC cell lines as well as normal renal tubular epithelial cells (HK2) (Figure 1a). We then tested if $A R$ might bind to non-coding RNAs (ncRNAs) to mediate its various functions. ${ }^{20-22}$ We used native RNA immunoprecipitation (RIP) assays with a

\footnotetext{
${ }^{1}$ Department of Urology, Shanghai Tenth People's Hospital, Tongji University, Shanghai 200072, China; ${ }^{2}$ Department of Urology, Renji Hospital, School of Medicine in Shanghai Jiao Tong University, 160 Pujian Road, Pudong District, Shanghai 200127, China; ${ }^{3}$ George Whipple Lab for Cancer Research, Departments of Pathology, Urology, Radiation Oncology and The Wilmot Cancer Center, University of Rochester Medical Center, Rochester, NY 14646 USA; ${ }^{4}$ Department of Urology and Chawnshang Chang Liver Cancer Center, Sir Run Run Shaw Hospital, Zhejiang University, Hangzhou 310016, China; ${ }^{5}$ Department of Pathology, Shanghai Tenth People's Hospital, Tongji University, Shanghai 200072, China and ${ }^{6}$ Sex Hormone Research Center, China Medical University/Hospital, Taichung 404, Taiwan

*Corresponding author: J Zheng, Department of Urology, Shanghai First People's Hospital, School of Medicine, Shanghai Jiao Tong University, Shanghai, China; and Department of Urology, Shanghai Tenth People's Hospital, Tongji University, Shanghai, China. E-mail: zhengjh0471@ @ina.com.cn

or C Chang, University of Rochester Medical Center, 601 Elmwood Avenue Rochester NY 14642, USA. Tel: 585 273 4500; Fax: 5857564133 ;

E-mail: chang@urmc.rochester.edu

${ }^{7}$ These authors contributed equally to this work.

Received 13.9.16; revised 28.3.17; accepted 04.4.17; Edited by E Baehrecke; published online 23.6.17
} 
a

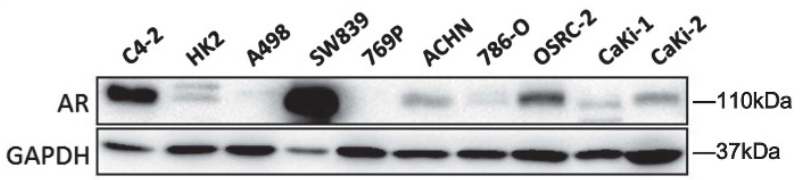

b

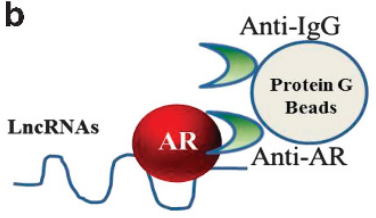

c

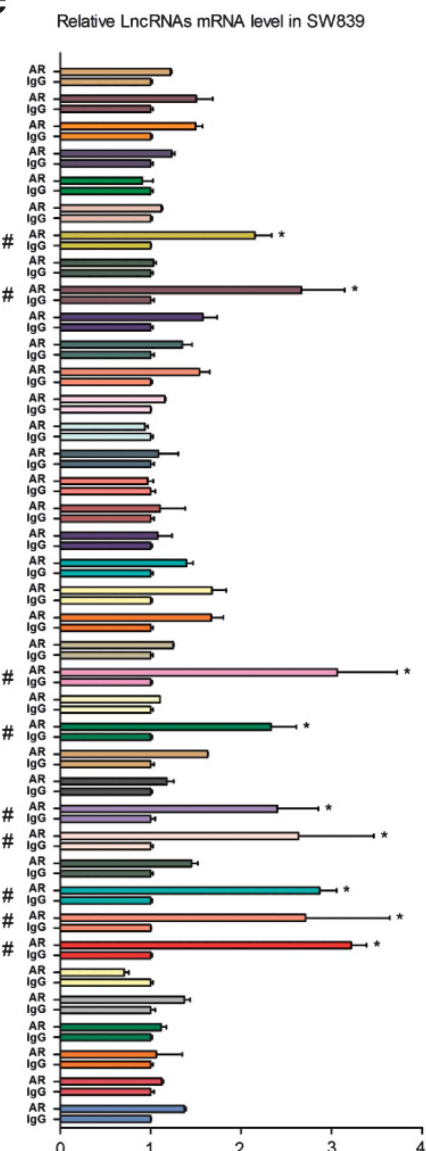

d

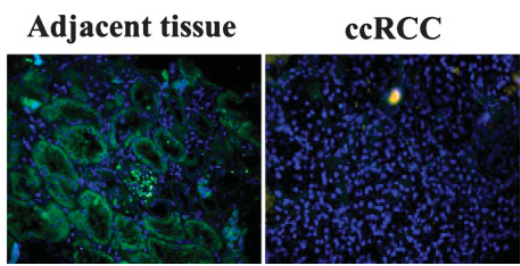

e

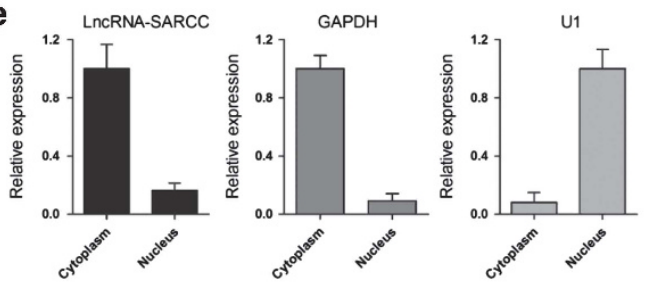

f
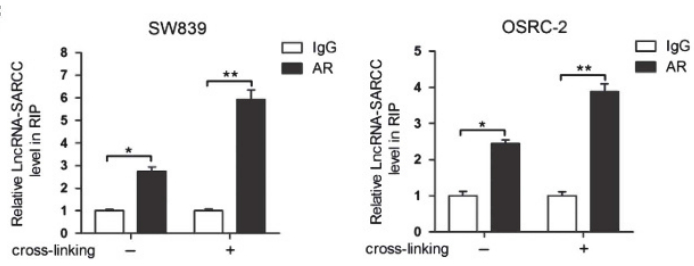

E ENST00000435496

ENSTO0000421314

ENSTO0000421118

口 ENST00000418694

口 ENST00000460407

$\square$ ENST00000458175

$\square$ ENST00000456816

口 ENST00000445535

口 ENST00000439042

$\square$ ENST00000420287

口 ENSTO0000412427

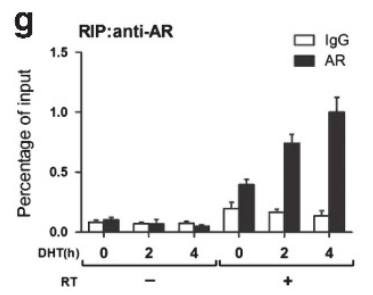

h

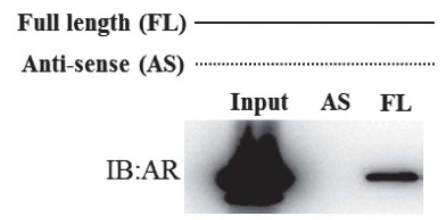

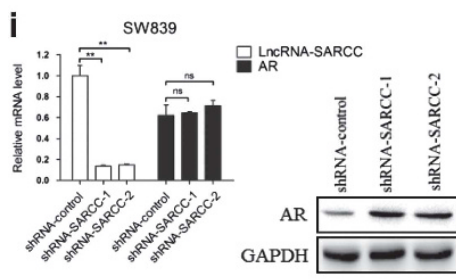

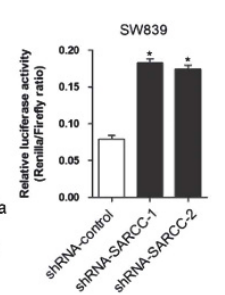

k
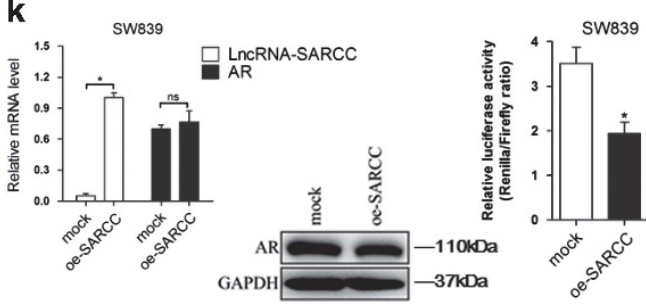

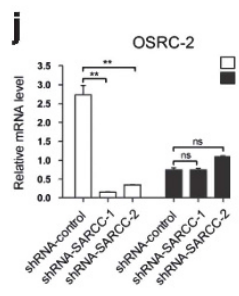

q LRCRNA-SARCC
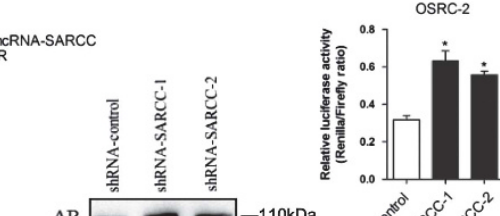

$\mathrm{AR}=-110 \mathrm{kDa}$ GAPDH $-37 \mathrm{KDa}$

Figure 1 For caption see page 1504

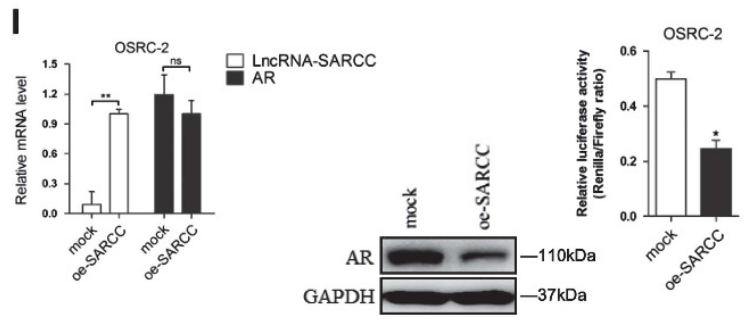


collection of deregulated ncRNAs identified in six RCC patients in a recently published paper, ${ }^{23}$ to examine potential AR-LncRNA interactions (Schematic Diagram, Figure 1b). We found that nine ncRNAs could potentially interact with AR in SW839 cells (Figure 1c). We then used OSRC-2 cells, another RCC cell line with less AR expression, to narrow down the candidate AR-interacting ncRNAs to five of them (Supplementary Figure S1A). In addition, A498 cells (AR non-detectable) were used to confirm that these five ncRNAs were undetectable in AR immunoprecipitates (data not shown).

Using Ensemble software, we further confirmed the characteristics of these five ncRNAs. Interestingly, only ENST00000460407 was confirmed to be a true LncRNA, whereas the others were processed pseudogenes (Supplementary Figures S1B and S1C). We named this LncRNA as SARCC (Suppressing Androgen Receptor in Renal Cell Carcinoma) based on later analyses. LncRNA-
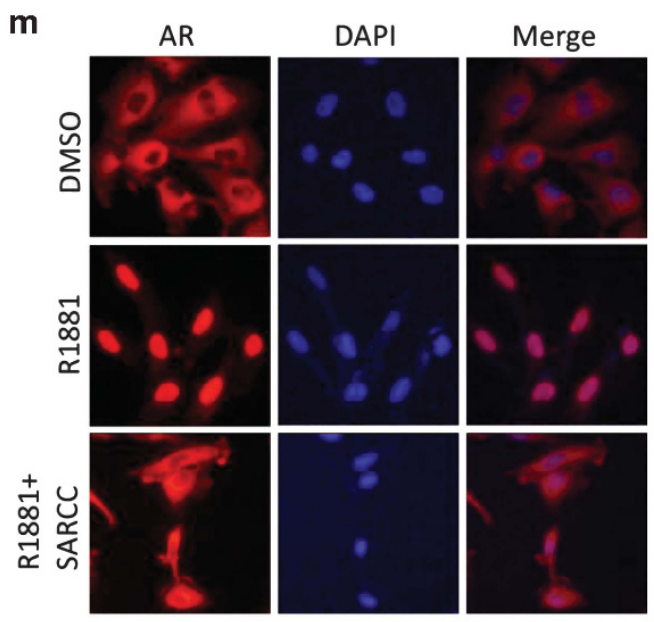

p

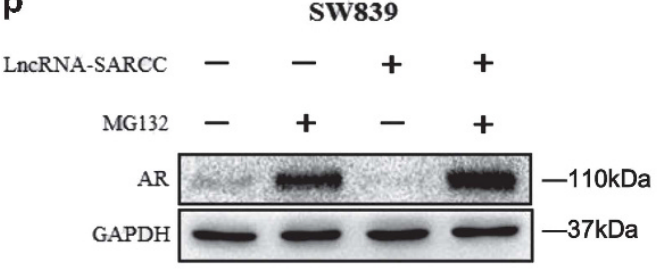

n
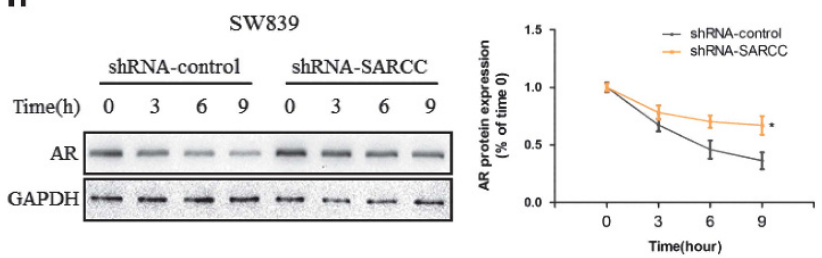

o
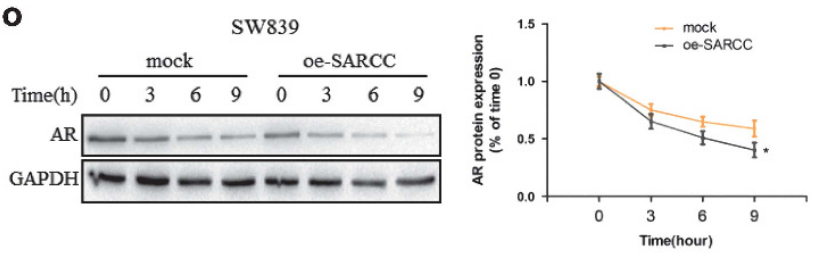

q

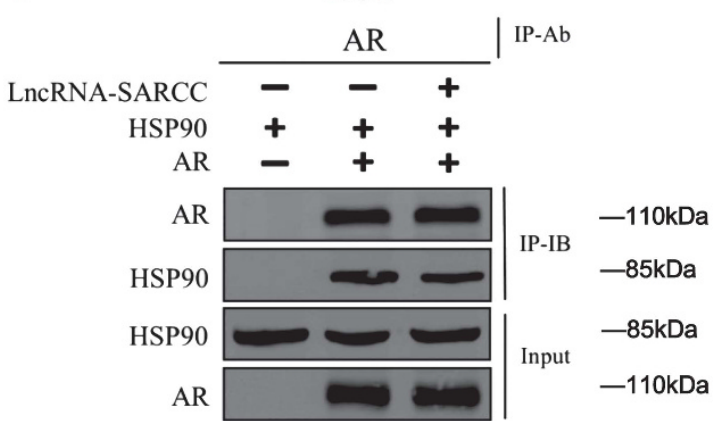

Figure 1 Continued

Figure 1 LnCRNA-SARCC is physically associated with and negatively correlated with AR. (a) Immunoblot of AR expression in a series of RCC cell lines or immortalized proximal tubule epithelial cell line from normal adult human kidney (HK2, A498, SW839, 769-P, ACHN, 786-O, OSRC-2, Caki-1 and Caki-2), with prostate cancer cell line C4-2 as positive control. (b) A schematic illustration of the procedure used to discover and define LncRNAs binding to AR in RCC tissues. (c) RIP assays for the potential LncRNA candidates endogenously associated with AR in SW839 cells. Total RNA was subjected to qRT-PCR assays. (d) Primary RCC and adjacent non-cancerous renal tissues were subjected to RNA FISH and analyzed by ultraviolet light excitation using a fluorescence microscope. (e) qRT-PCR for LncRNA-SARCC, GAPDH and U1 from RNA extracted from cytoplasmic and nuclear fractions. (f) SW839 and OSRC-2 cells were cross-linked with/without $4 \%$ paraformaldehyde before RIP assays were carried out. (g) RIP assay in $10 \mathrm{nM}$ DHT-treated SW839 cells at the indicated time points. (h) RNAs corresponding to different fragments of LncRNA-SARCC were biotinylated and incubated with SW839 cell extracts, targeted with streptavidin beads and washed. Associated AR protein was detected by WB and compared between full length LncRNA-SARCC and its antisense RNA. (i and j) qRT-PCR assays (left panels) for the shRNA-SARCC mRNA level in stable SW839 (i) and OSRC-2 (j) cell clones. AR protein and luciferase level were measured by WB (middle panels) and luciferase reporter assay (right panels). (k and I) qRT-PCR assays for the oe-SARCC mRNA levels in stable SW839 (k) and OSRC-2 (I) cell clones. AR protein and luciferase level were measured by WB (middle panels) and luciferase reporter assay (right panels). (m) Immunofluorescence staining of AR. SW839-control and SW839-

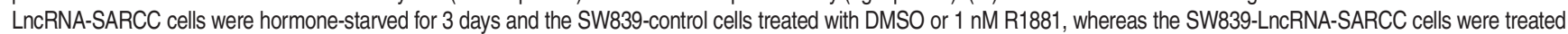
with R1881 for $24 \mathrm{~h}$ before subjected to immunostaining using an anti-AR antibody. (n) SW839 cells expressing control shRNA or LncRNA-SARCC shRNA were treated with $20 \mathrm{mg} / \mathrm{ml}$ cycloheximide (CHX) for the indicated time periods and cell lysates analyzed by WB. (o) SW839 cells expressing mock or oe-LncRNA-SARCC were treated as in (n) and analyzed by

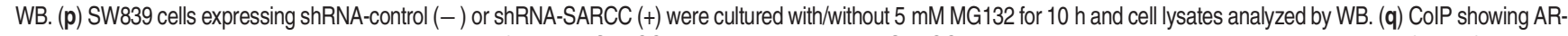
Hsp90 protein interaction with the absence or presence of LncRNA-SARCC. AR, Hsp90 and LncRNA-SARCC were expressed in 293T cells through transient transfection followed by AR immunoprecipitation. AR and associated Hsp90 protein was detected by immunoblot analysis. Data shown are mean \pm S.D. $(n=3)$. ${ }^{\star} P<0.05,{ }^{* \star} P<0.01$ 
a
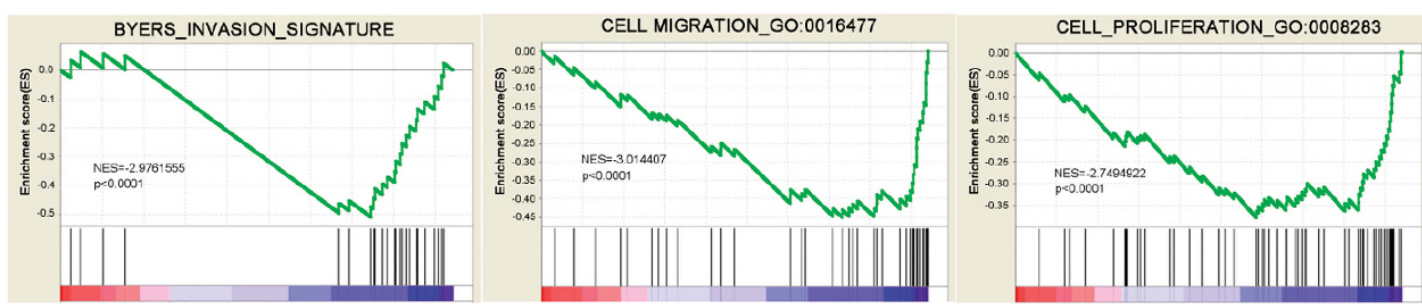

b
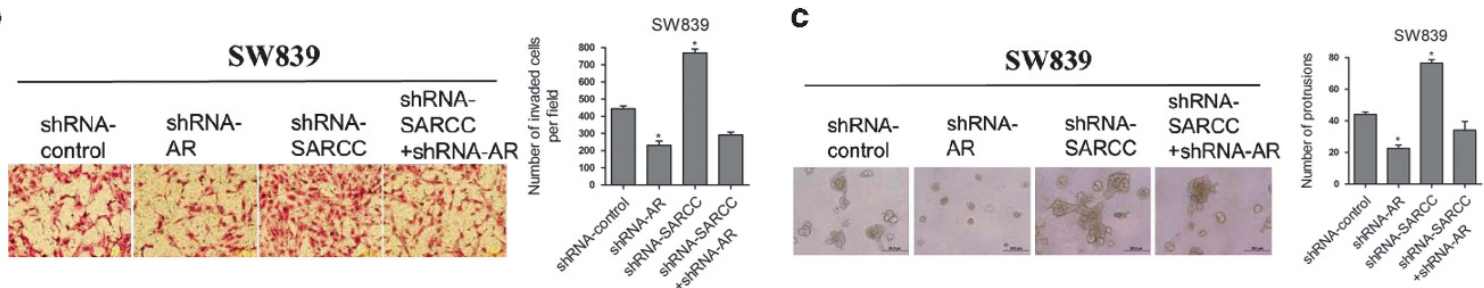

d

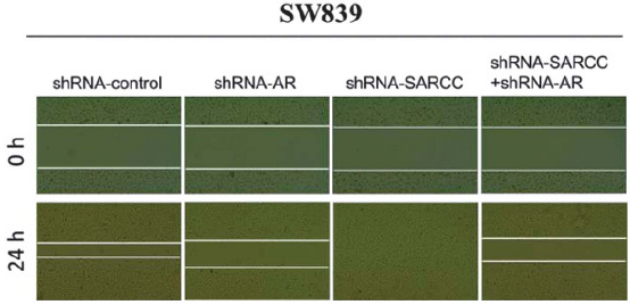

f

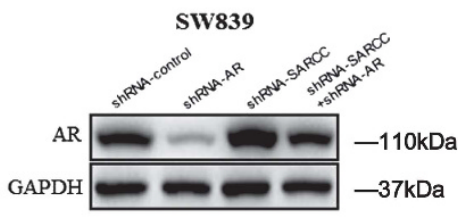

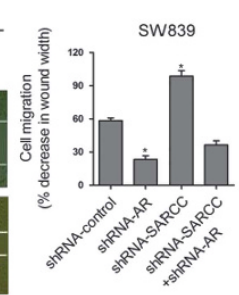

e

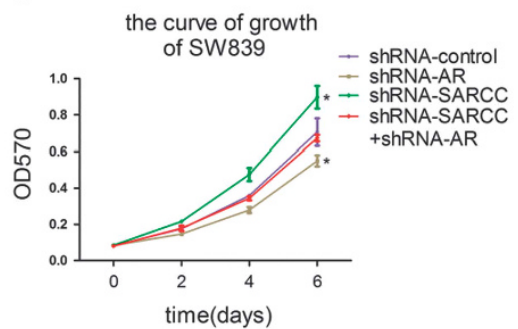

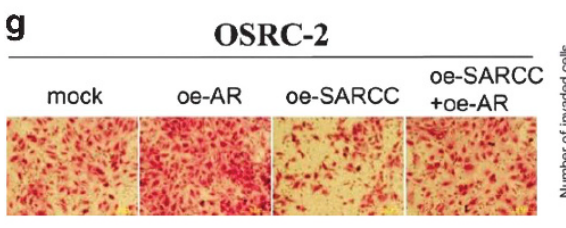

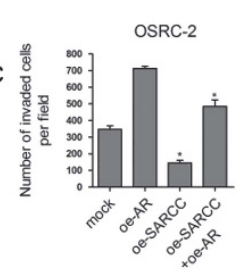

h
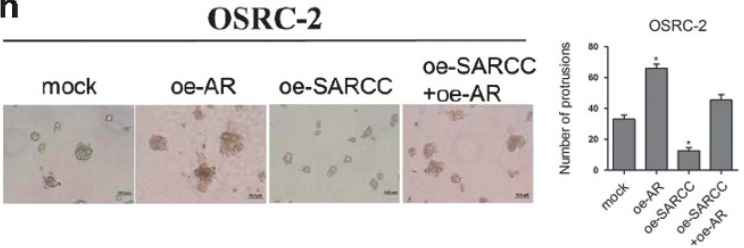

i

OSRC-2
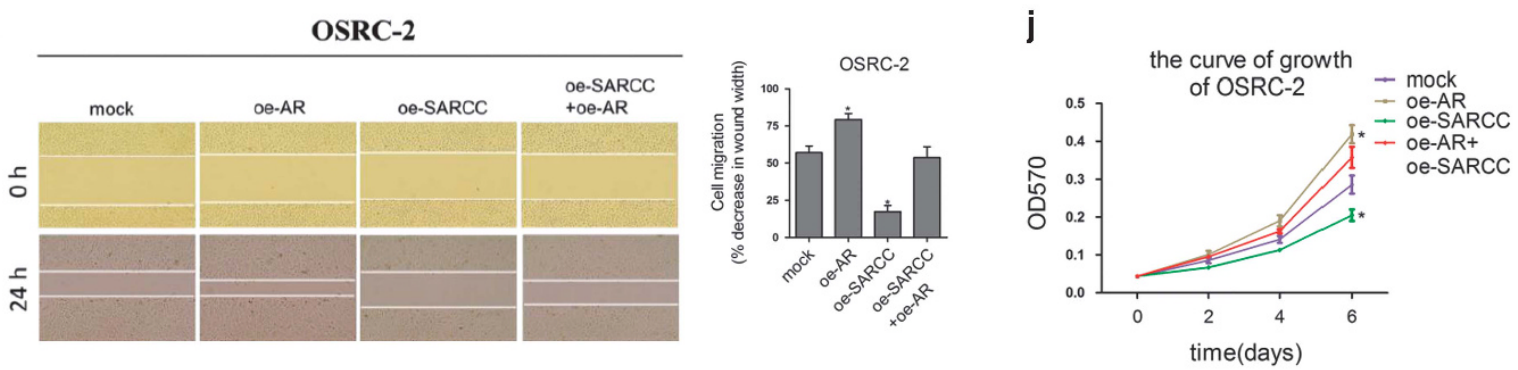

k

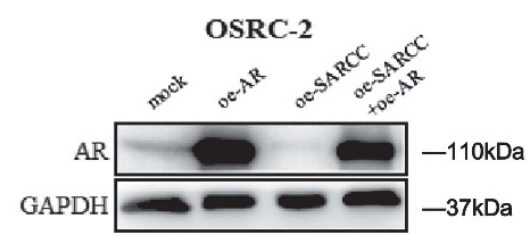

Figure 2 For caption see page 1506 
SARCC has little protein-coding potential based on PhyloSCF of -236.4277 (Supplementary Figure S1D). ${ }^{24}$ Furthermore, LncRNA-SARCC was mainly located in the cytoplasm from RNA Fluorescent in situ hybridization (FISH) on renal cancer tissues and subcellular fraction assay (Figures $1 \mathrm{~d}$ and $\mathrm{e}$ ).

RIP assay in the presence or absence of cross-linker with $4 \%$ paraformaldehyde further supported the interaction between LncRNA-SARCC with AR in both AR-positive cell lines (SW839 and OSRC-2) (Figure 1f). Furthermore, dihydrotestosterone (DHT) also enhanced the interaction of LncRNA-SARCC with AR in SW839 cells (Figure 1g). It was possible that DHT stimulated LncRNA-SARCC expression in a dose- and time-dependent manner, thus promoting the interaction between AR and LncRNA-SARCC (Supplementary Figures S1E and S1F). Consistent with this, when AR expression was reduced through RNA interference, LncRNA-SARCC level was reduced in SW839/shRNA-AR cells (Supplementary Figure S1G). More significantly, binding assays using in vitro-synthesized biotin-labeled LncRNASARCC mixed with RCC cell lysates also proved that AR could directly bind to LncRNA-SARCC (Figure 1h). In a reciprocal manner, knocking down LncRNA-SARCC (shRNA-SARCC) in SW839 and OSRC-2 cells enhanced AR protein, but not mRNA expression as well as AR activity through luciferase reporter assay (Figures $1 \mathrm{i}$ and $\mathrm{j}$ ). The mRNA expression of FKBP5 and TMPRSS2, two key AR target genes, was also increased by shRNA-SARCC (Supplementary Figures $\mathrm{S} 1 \mathrm{H}$ and S1I). Consistent with these findings, overexpressing LncRNA-SARCC (oe-SARCC) resulted in reduction of $A R$ expression, transactivation and AR target gene expression (Figures $1 \mathrm{k}$ and I, Supplementary S1J and S1K). In addition, AR immunofluorescence staining in the androgen-deprived or stimulated control and LncRNASARCC-expressing SW839 cells demonstrated that oe-SARCC prevented AR movement from the cytoplasm to the nucleus (Figure $1 \mathrm{~m}$ ).

We applied cycloheximide, an inhibitor of de novo protein synthesis in eukaryotic cells, ${ }^{25}$ and found that shRNA-SARCC enhanced the stability of AR protein (Figure $1 \mathrm{n}$ and Supplementary Figure S1L), whereas oe-SARCC suppressed the stability of AR protein (Figure 10 and Supplementary Figure $\mathrm{S} 1 \mathrm{M}$ ). In addition, AR protein induction was restored by the proteasome inhibitor MG132, indicating that AR protein was decreased by LncRNA-SARCC in a proteasome-dependent manner (Figure $1 p$ and Supplementary Figure S1N). Previous studies demonstrated that heat shock protein 90 (HSP90) had a key role in androgen-induced nuclear localization and activation of $\mathrm{AR}^{26,27}$ We thus hypothesized that LnCRNA-SARCC binding with the AR protein prevented AR from interacting with HSP90. To test this, we co-transfected 293T cells with HSP90 and with/without AR and LnCRNA-SARCC. The immunoprecipitation followed by western blot of AR protein indicated that HSP90 physically interacted with AR, which could be inhibited by LncRNASARCC (Figure 1q).

Figure 3 LncRNA-SARCC-AR signals went through miR-143-3p. (a) qRT-PCR assays for the 16 miRNAs in SW839 cells transfected with shRNA-AR versus shRNA-control group and oe-SARCC versus mock group. Three out of 16 miRNAs were significantly increased ( $\geqslant 0.3$ fold) in the shRNA-AR group and oe-SARCC group. (b) qRT-PCR assays for the three miRNAs in OCRC-2 cells transfected with shRNA-SARCC versus shRNA-control (left panel) or oe-AR versus mock (right panel). (c, d) The miR-143-3p expression measured by qRT-PCR assays in SW839 cells (c) expressing shRNA-control, shRNA-AR, shRNA-SARCC and shRNA-AR + shRNA-SARCC and in OSRC-2 cells (d) expressing mock, oe-AR, oe-SARCC and oe-AR + oe-SARCC. (e) Bioinformatic analysis of potential AR binding sites in miR-143-3p promoter. (f) Lysates of SW839 cells were subjected to ChIP assay and amplified by PCR reaction. (g) A schematic illustration of ARE1-mut and ARE2-mut in the miR-143-3p promoter. (h-i) HEK293T (h) and SW839 (i) cells were cotransfected with the indicated reporter constructs and Renilla luciferase plasmid. After 48 hours transfection, reporter activity was then measured and plotted after normalizing with Renilla luciferase activity. (j) Number of invaded cells per high-power field (left), wound-healing assay (middle) and MTT proliferation change (right) as induced by the transfection of oe-miR-143-3p with shRNA-SARCC SW839 cells versus shRNA-control cells. (k) Number of invaded cells per high-power field (left), wound-healing assay (middle) and MTT proliferation change (right) in OSRC-2 cells with the transfection of oe-miR-143-3p in shRNA-SARCC OSRC-2 cells versus shRNA-control cells. (i) GSE53757 data set indicated the relationship between LncRNA-SARCC and miR-143-3p. (m) Western blot analysis for miR-143-3p target genes AKT, MMP-13, K-RAS and P-ERK protein levels with miR-143-3p mimic and inhibitor in SW839 cells. (n) Results of luciferase reporter assay. SW839 and OSRC-2 cell lines infected with miR-143-3p mimic or vector control lentivirus were co-transfected with pmirGLO vector containing the WT sites of the K-RAS, MMP-13 and AKT of 3'UTR. (o) GSE53757 data set indicated the relationship between miR-143$3 p$ and target genes K-RAS, MMP-13 and AKT. (p) Western blot analysis for AKT, MMP-13, K-RAS and P-ERK protein levels of shRNA-SARCC in SW839 (upper) and OSRC-2 (lower) cells. These effects were abolished by transfection of oe-miR-143-3p in SW839 and OSRC-2 cells. Data shown are mean \pm S.D. $(n=3) .{ }^{*} P<0.05$

Figure 2 LncRNA-SARCC suppressed RCC cell progression through AR. (a) GSEA of BYERS, GO:0016477 and G0:0008283 databases referred to invasion, migration and proliferation related-gene signatures, respectively, of LnCRNA-SARCC in low-grade versus high-grade RCC tissues. NES, normalized enrichment score. (b) Representative images (left panel) and the numbers of invasive cells per high-power field (right panel) induced by the transfection of shRNA-SARCC in SW839 cells versus shRNA-control cells. Transfection of shRNA-SARCC restored the invasive capabilities of shRNA-AR in SW839 cells. (c) Representative micrographs (left panels) and number of cells grown on matrigel for 8 days in 3D spheroid invasion assay (right panel) for SW839 cells with shRNA-SARCC or shRNA-control. The shRNA-SARCC restored the invasive capabilities of shRNA-AR in SW839 cells. (d) Representative micrographs of wound-healing assay (left panel) and number of cells (right panel) for SW839 cells with shRNA-SARCC versus shRNA-control. The shRNA-SARCC reversed the effect of shRNA-AR on cell migration in SW839 cells. Wound closures were photographed at 0 and $24 \mathrm{~h}$ after wounding. (e) MTT proliferation change for SW839 cells with shRNA-SARCC versus shRNA-control. The shRNA-AR reduced the growth of shRNA-SARCC SW839 cells. (f) Western blot analysis shows the transfection of shRNA-AR restored the upregulation of AR induced by stable shRNA-SARCC expression in SW839 cells. (g) Representative images (left panel) and number of invasive cells per high-power field (right panel) was reduced by the transfection of oe-SARCC in OSRC-2 cells versus mock cells. (h) Representative micrographs (left panel) and number of cells grown on matrigel for 8 days in 3D spheroid invasion assay (right panel) after transfection of oe-SARCC in OSRC-2 cells versus mock cells. (i) Representative micrographs (left panel) of wound-healing assay and number of cells (right panel) after transfection of oe-SARCC in OSRC-2 cells compared with mock cells. Wound closures were photographed at 0 and $24 \mathrm{~h}$ after wounding. (j) MTT proliferation change with oe-SARCC compared with mock in OSRC-2 cells. Transfection of oe-AR restored the growth of oe-SARCC in OSRC-2 cells. (k): Western blot analysis in OSRC-2 cells with oe-AR and oe-SARCC. For $\mathbf{e}$ and j, data shown are means \pm S.D. ${ }^{\star} P<0.05$ 
a

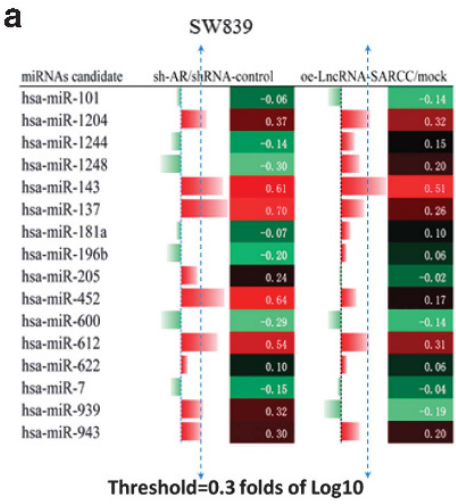

e

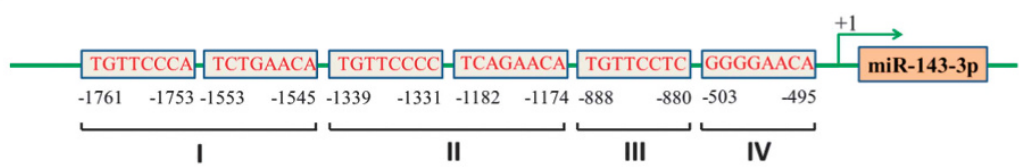

b
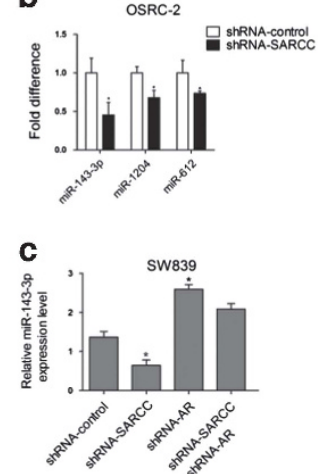

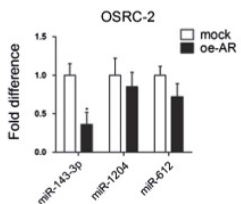

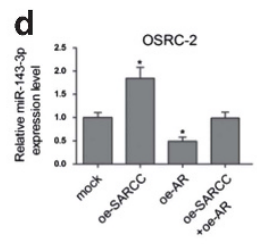

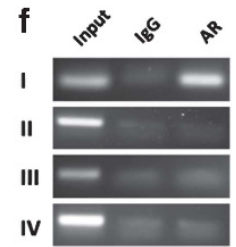

g

\begin{tabular}{|l|l|l|}
\hline wt: TGTTCCCA & TCTGAACA \\
mut: TGCCCTTA & TCCAAGTA \\
\hline
\end{tabular}
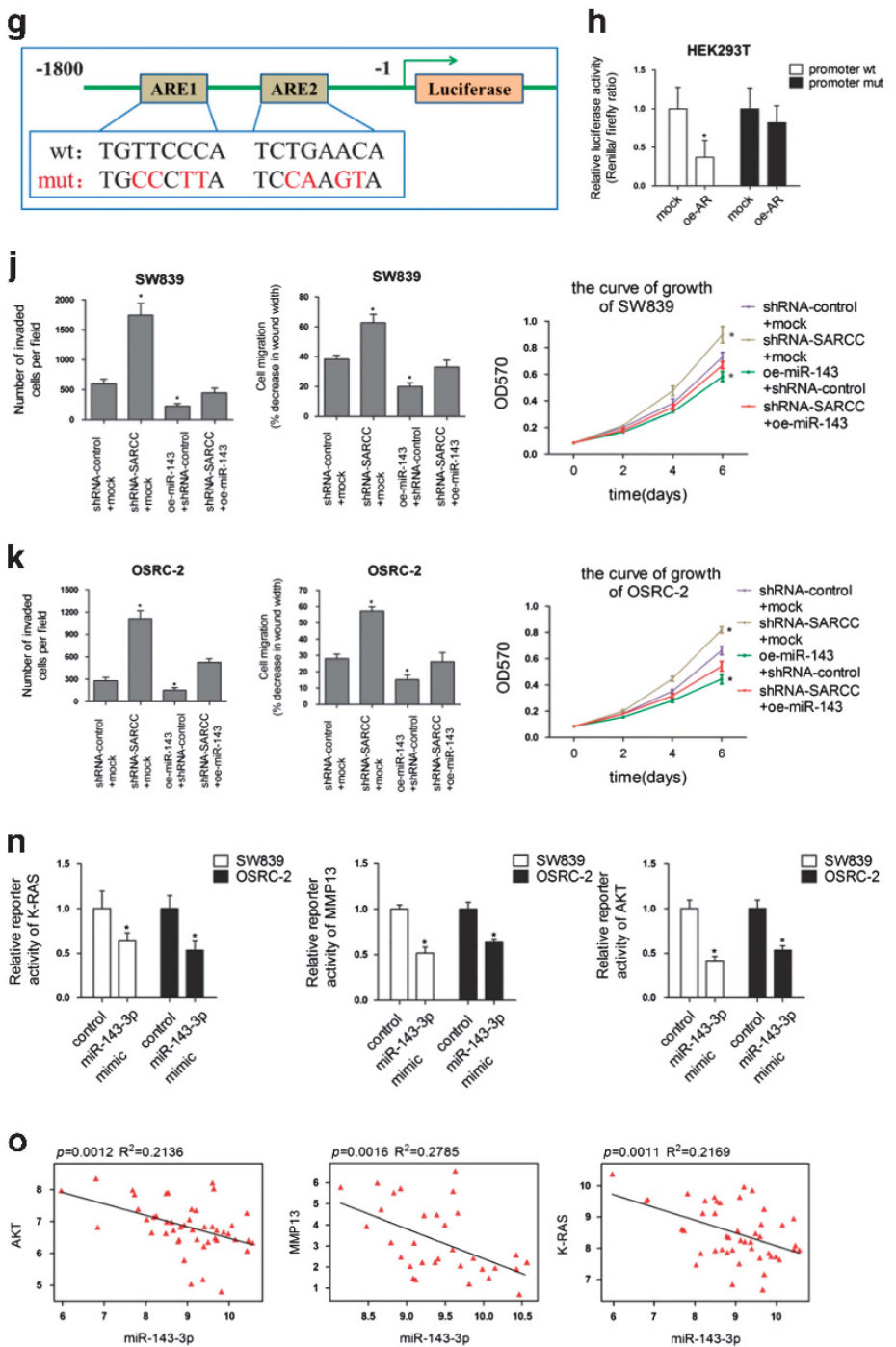
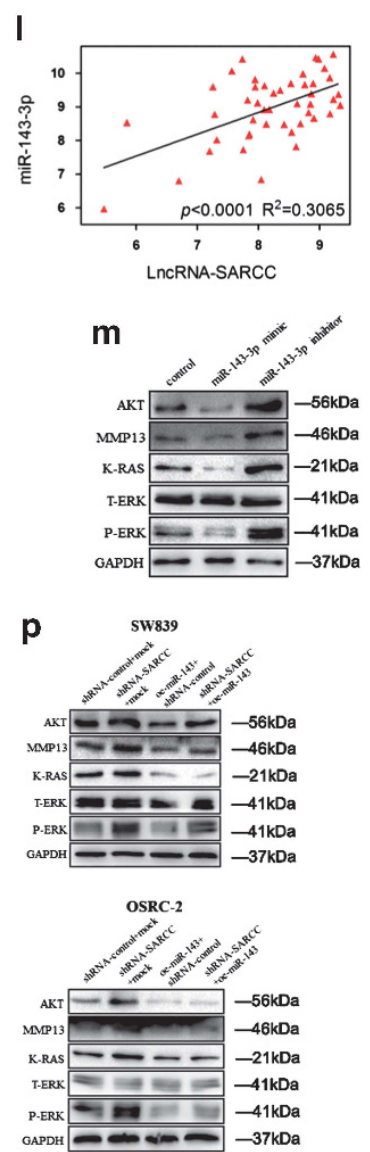
Together, results from Figure 1 and Supplementary Figure S1 demonstrated that LncRNA-SARCC could directly bind to $A R$ and destabilize AR protein.

LncRNA-SARCC suppressed RCC cell progression through AR. To further examine whether LncRNA-SARCC possesses tumor-suppressive properties, we performed gene set enrichment analysis to link the published gene array analysis of clear cell RCC (ccRCC) versus matched normal kidney tissue signatures (GEO Datasets: GSE53757; Genesets: GO:0016477, GO:0008283 and $B Y E R S^{28}$ ), and results revealed that LncRNA-SARCC expression was negatively related with $\mathrm{RCC}$ cell invasion, migration and proliferation (Figure 2a).

The shRNA-SARCC in both SW839 and OSRC-2 cells resulted in altered morphology, such as a scattered, not clustered, distribution of cells as well as a spindle-shaped contour (Supplementary Figure S2A). Using matrigel-coated chamber invasion assays and 3D culture invasion assays, we observed that shRNA-SARCC markedly increased the invasiveness of SW839 cells (Figures $2 b$ and c). Wound-healing migration assay and MTT assay also demonstrated that shRNA-SARCC promoted the migratory speed and cell proliferation (Figures $2 d$ and e). Importantly, an interruption approach with shRNA-AR reversed the effects of shRNASARCC on cell invasion, migration and proliferation (Figures $2 b-e)$. As expected, WB confirmed that shRNA-AR partially blocked the effect of induced AR protein caused by shRNASARCC in SW839 cells (Figure 2f).

In contrast, LncRNA-SARCC overexpression (oe-SARCC) in OSRC-2 cells attenuated cell invasion, migration and proliferation, whereas the interruption approach using $A R$ overexpression also partially reversed these changes (Figures $2 g-j)$. WB revealed that oe-AR partially inhibited the effect of reduced $A R$ protein in OSRC-2/oe-SARCC cells (Figure $2 k$ ). Importantly, shRNA-SARCC failed to enhance RCC cell invasion, migration and growth in 786-O and A498 cells as they appeared to be negative for AR expression (Supplementary Figure S2B-S2D).

Together, results from Figure 2 and Supplementary Figure S2 demonstrated that LncRNA-SARCC suppressed RCC progression by modulating AR.

LncRNA-SARCC-AR signals went through miR-143-3p. Accumulating evidence indicated that miRNAs are frequently deregulated in various human urinary tract tumors and have multiple functions in biological processes. ${ }^{29-31}$ To dissect the molecular mechanism how LncSARCC/AR interaction alters the RCC progression, we focused on the role of miRNAs in these processes. We used gene co-expression networks to cluster the transcripts into phenotypically relevant coexpression modules through the GSE53757 database and explored 16 miRNAs that have been connected with both LnCRNA-SARCC and AR, 9 miRNAs related to LncRNASARCC, and 9 miRNAs related to AR (Supplementary Figures S3A and S3B). We applied qRT-PCR assays in SW839 cells with shRNA-AR or oe-SARCC to test if any of those miRNAs might be responsive to LncRNA-SARCC-AR expression. As shown in Figure $3 a$ and Supplementary Figure S3C miRNAs (miR-143-3p, miR-1204 and miR-612) were significantly enhanced ( $\geqslant 0.3$ fold of Log10) by shRNAAR or oe-SARCC. We used OSRC-2 cells to determine their response through oe-AR or shRNA-SARCC. The results indicated that miR-143-3p has a more significant decrease in both shRNA-SARCC group and oe-AR group (Figure 3b).

To further confirm LncRNA-SARCC increased miR-143-3p expression via regulating $A R$, we knocked down LncRNASARCC and found it led to a reduction of miR-143-3p expression (Figure 3c). Importantly, shRNA-SARCC-reduced miR-143-3p expression was partially reversed by knocking down AR in SW839 cells (Figure 3c). Consistently, oe-SARCC induced miR-143-3p expression, which could be reversed by oe-AR in OSRC-2 cells (Figure $3 d$ ).

It was recognized that $A R$, acting as a transcriptional factor, might directly regulate the transcription of certain miRNAs. ${ }^{32}$ We hypothesized that AR could directly decrease miR-143-3p expression by binding to the potential androgen response elements (AREs) in its promoter. We applied miRStart to determine the transcriptional start site (TSS) of the precursor of miR-143, pre-miR-143, as well as potential AREs within 2000 bases upstream from the TSS. The results suggested that this potential promoter region might contain four AREs (Figure $3 e$ ). ChIP assay with anti-AR antibody confirmed that AR could bind to the ARE located between - 1761 and - 1545 nucleotides in the promoter of miR-143-3p (Figure 3f). Furthermore, AR decreased miR-143-3p promoter activity but not that with the ARE-mut in HEK293T cells (Figures $3 \mathrm{~g}$ and h). Conversely, shRNA-AR increased miR-143-3p promoter activity but failed to do so with ARE-mut in SW839 cells (Figure 3i).

Figure 4 LncRNA-SARCC was inversely correlated with AR, AKT, MMP-13, K-RAS and P-ERK in ccRCC tissues. (a) LncRNA-SARCC expression in human RCC tissues and paired adjacent non-cancerous renal tissues. (b) Relative LnCRNA-SARCC expression levels in RCC tumors are presented as fold change $=2^{\text {( } \triangle \mathrm{Ct} \text { normal }-\triangle \mathrm{Ct} \text { tumor) }}$ of tumor versus matched normal tissues. The 0.5 -fold change threshold was defined as differentially expressed. (c) IHC staining revealed the location of AR expression in ccRCC specimens $(\times 200, \times 400)$ versus adjacent normal tissues. (d) The correlation between LncRNA-SARCC expression and AR protein levels in ccRCC tissues. (e) LncRNA-SARCC expression in a series of RCC cell lines (A498, SW839, 769-P, ACHN, 786-O, OSRC-2, Caki-1 and Caki-2). For (a) and (e), the expression level of LncRNA-SARCC were analyzed by qRT-PCR. (f) LncRNA-SARCC expression in metastatic (meta) ccRCC tissues and non-meta ccRCC tissues. (g) The expression of AR protein was determined by western blot analysis in eight pairs of metastatic ccRCC tissues (M1-M8) and non-metastatic ccRCC tissues (N1-N8). (h-k) Immunohistochemical staining revealed the location of AKT (h), MMP-13 (i), K-RAS (j) or P-ERK (k) expression in ccRCC specimens $(\times 200, \times 400)$. Two representative cases are shown. (I) The correlation between LncRNASARCC expression and AKT, MMP-13, K-RAS or P-ERK protein levels in cCRCC tissues. ( $m$ ) Kaplan-Meier analyses of the correlations between LncRNA-SARCC expression level and overall survival of 66 patients with RCC. The median expression level was used as the cutoff. Patients with LncRNA-SARCC expression values below the 50 th percentile were classified as having lower LncRNA-SARCC levels. Patients with LncRNA-SARCC expression values above the 50th percentile were classified as having higher LncRNASARCC levels. The horizontal lines in the box plots represent the medians, the boxes represent the interquartile range, and the whiskers represent the 2.0th and 97.5 th percentiles. The significant differences between samples were analyzed using the Wilcoxon signed-rank test 
Intriguingly, early reports indicated that the miR-143 and miR-145 cluster shared a common promoter that has been linked to a potential strong tumor suppressor in some selective tumors. ${ }^{33,34}$ Here we found shRNA-SARCC or oe-SARCC slightly changed miR-145 expression compared with the altered miR-143 expression in SW839 and OSRC-2 cells (Supplementary Figures S3D and S3E), indicating that some other co-regulators might be responsible for this differential regulation of $\mathrm{miR}-143$ and miR-145.

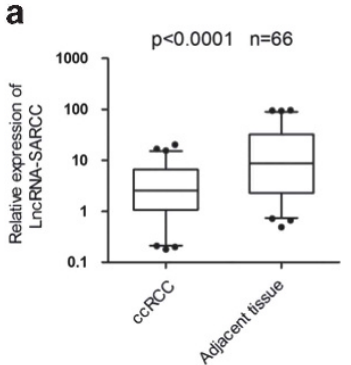

b

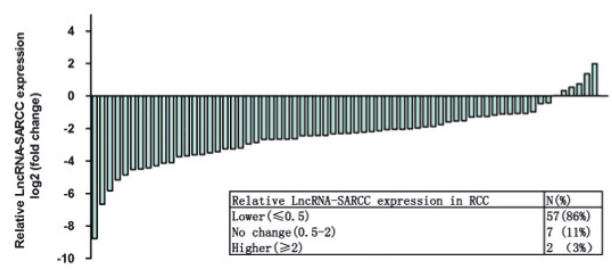

d

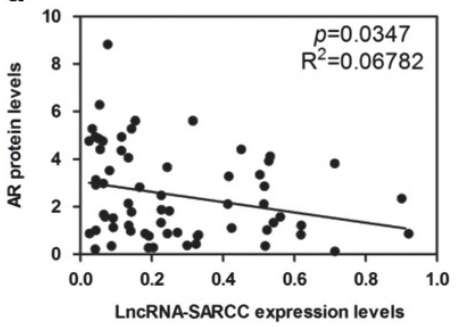

e

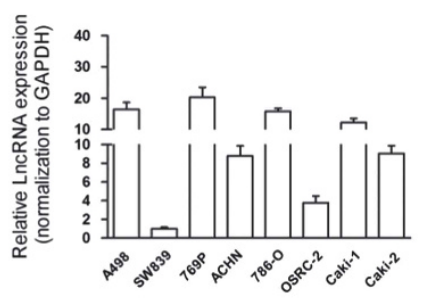

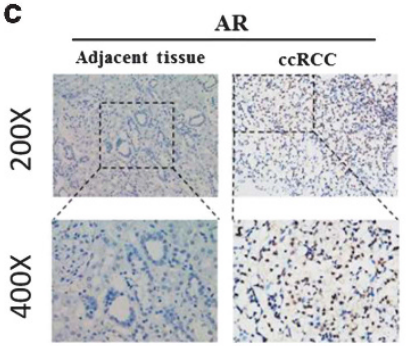

f

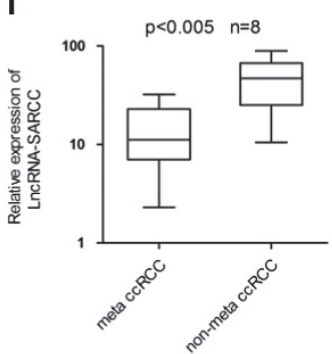

g $\begin{array}{llllllllllllllll}\mathrm{N} 1 & \mathrm{M} 1 & \mathrm{~N} 2 & \mathrm{M} 2 & \mathrm{~N} 3 & \mathrm{M} 3 & \mathrm{~N} 4 & \mathrm{M} 4 & \mathrm{~N} 5 & \mathrm{M} 5 & \mathrm{~N} 6 & \mathrm{M} 6 & \mathrm{~N} 7 & \mathrm{M} 7 & \mathrm{~N} 8 & \mathrm{M} 8\end{array}$

AR $110 \mathrm{kDa}$

\section{ACTIN}

h
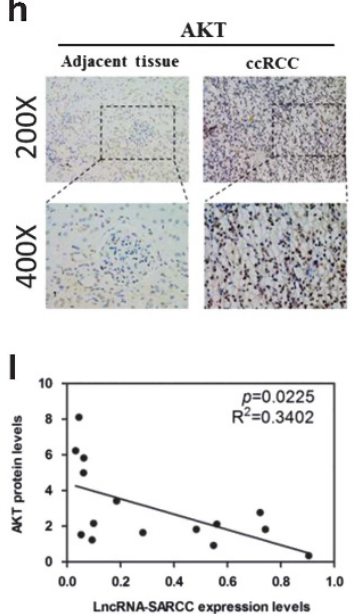
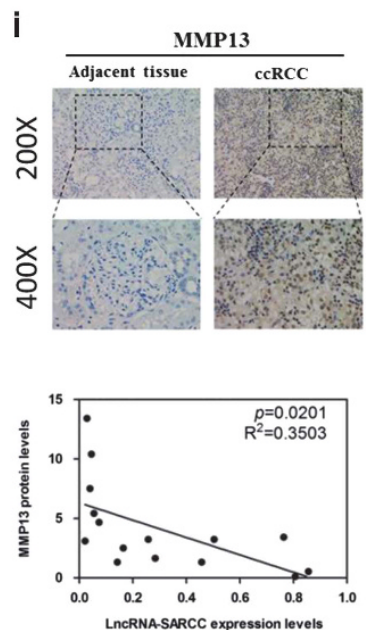
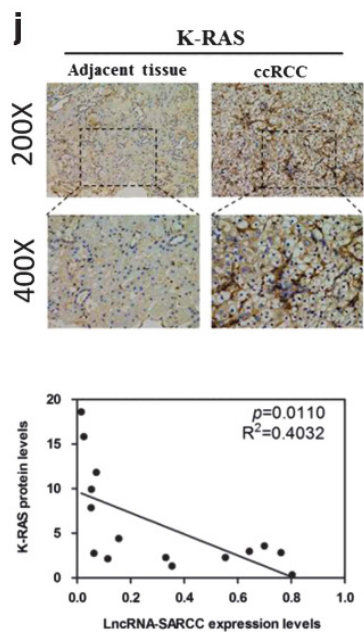

$\mathbf{k}$
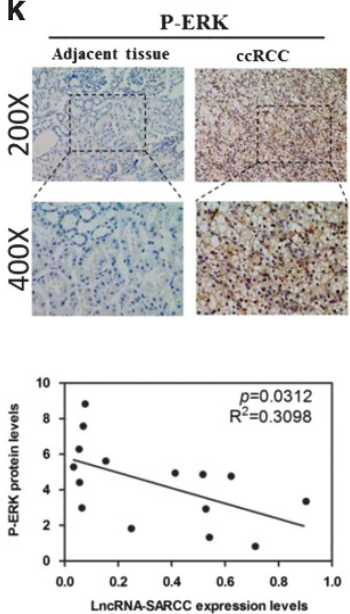

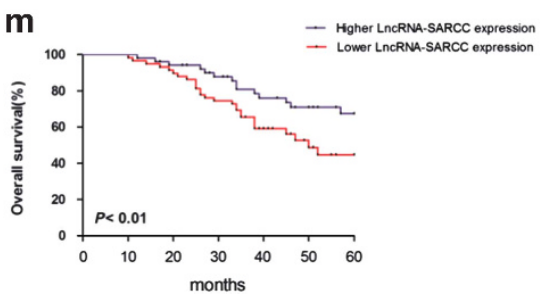


Importantly, miR-143-3p overexpression partially abolished the effect of knocked down LncRNA-SARCC expression in SW839 cells in regulating RCC cell invasion, migration and proliferation (Figure 3j). Similarly, overexpression of miR-143-3p in OSRC-2 cells blocked the effect of invasion, migration and proliferation from LncRNA-SARCC knockdown (Figure 3k). Furthermore, correlation analysis in GSE53757 data set supported that LncRNA-SARCC and miR-143-3p had a positive relationship in $46 \mathrm{RCC}$ tissues (Figure $3 \mathrm{I}$ ).

To determine the potential target genes of miR-143-3p for regulating $\mathrm{RCC}$ progression, using different miRNA bioinformatic softwares, we found miR-143-3p sites at the 3'-UTR of AKT, MMP-13 and K-RAS as reported in published literatures (Supplementary Figure S3F). ${ }^{34-38}$ We then used the miR-143$3 p$ mimic and miR-143-3p inhibitor to examine its effects on the potential target gene expression. As shown in Figure $3 \mathrm{~m}$, the protein levels of AKT, MMP-13, K-RAS and phospho-ERK (P-ERK) were significantly enhanced in response to miR-143$3 p$ inhibitor, whereas suppressed by miR-143-3p mimic in SW839 cells. Notably, the miR-143-3p mimic inhibited the luciferase activity of WT 3'-UTR of K-RAS, MMP-13 and AKT (Figure 3n). Similarly, GSE53757 data set supported that miR-143-3p was negatively correlated with K-RAS, MMP-13 and AKT mRNA levels (Figure 3o).

Next, we explored whether LncRNA-SARCC could regulate miR-143-3p target genes. The oe-SARCC significantly decreased AKT, MMP-13, K-RAS and P-ERK protein levels (Supplementary Figure S3G), whereas shRNA-SARCC effectively increased AKT, MMP-13, K-RAS and P-ERK protein levels in both cell lines (Supplementary Figures $\mathrm{S} 3 \mathrm{H}$ ). In addition, the rescue experiment indicated that oe-miR-143-3p could partially negate the effect of increased AKT, MMP-13, K-RAS and P-ERK protein levels caused by shRNA-SARCC in both cell lines (Figure 3p).

Together, these results suggested that LncRNA-SARCC could inhibit RCC progression through modulation of the miR-143-3p-mediated AKT, MMP-13, K-RAS and P-ERK signals.

LncRNA-SARCC was inversely correlated with AR, AKT, MMP-13, K-RAS and P-ERK in ccRCC tissues. To further verify the LncRNA-SARCC expression in human CCRCC, we used qRT-PCR to examine 66 paired primary $\mathrm{CCRCC}$ and adjacent normal tissues. The results revealed that RNA levels of LncRNA-SARCC were much lower in $86 \%(57 / 66)$ of ccRCC tissues $(P<0.0001)$ than in the paired non-cancerous renal tissues (Figures $4 \mathrm{a}$ and b). RNA FISH assay of Figure 1d demonstrated detectable LncRNA-SARCC expression in non-cancerous renal tissues but undetectable LncRNA-SARCC expression in paired renal cancer tissues. Interestingly, we performed RNA FISH assay to visualize
LncRNA-SARCC expression in other human tissues, and found it was not expressed in various types of normal and cancer tissues, such as colon, gastric, prostate and bladder (Supplementary Figure S4A). Also, LncRNA-SARCC expression was decreased in liver cancers, compared with normal tissues (Supplementary Figure S4A). These results indicated that LncRNA-SARCC might be a tissue-specific ncRNA in human organs and correlated with disease development.

Immunohistochemical staining of AR expression in ccRCC tissues indicated a higher expression than in adjacent normal tissues (Figure 4c). LncRNA-SARCC expression was negatively related with AR protein expression in ccRCC samples $(P=0.0347)$ (Figure $4 d)$. Meanwhile, we validated several analyses of ccRCC array data (Gumz Renal Statistics ( $n=20$, $P<0.05)$; Yusenko Renal Statistics $(n=67, P<0.05)$; Jones Renal Statistics $(n=92, P<0.05)$; Higgins Renal Statistics ( $n=44, P<0.05$ ) supporting our AR IHC staining through Oncomine analysis (Supplementary Figures S4B-S4E). ${ }^{39-42}$ An inverse correlation of LncRNA-SARCC with AR expression was observed by comparing both LncRNA-SARCC level and $A R$ protein level in various RCC cell lines (Figures 1a and $4 \mathrm{e}$ ). Furthermore, the expression of LncRNA-SARCC was significantly lower in metastatic cCRCC tissues than that of pairmatched non-metastatic ccRCC tissues, whereas AR was markedly higher in metastatic tissues (M) than that of nonmetastatic tissues ( $N$ ) by WB analysis (Figures $4 \mathrm{f}$ and $\mathrm{g}$ ). In addition, IHC staining proved that AKT, MMP-13, K-RAS and $P$-ERK expressions were higher in CCRCC tissues than in adjacent normal tissues (Figures $4 \mathrm{~h}-\mathrm{k}$ ), which were negatively related with LncRNA-SARCC expression in human cCRCC (Figure 4I).

We next examined the relationship between LncRNASARCC expression levels and the clinical-pathological characteristics of the $66 \mathrm{ccRCC}$ samples (Supplementary Table 1). Correlation regression analysis revealed that low expression of LncRNA-SARCC was obviously correlated with tumor size $(P=0.012)$, Fuhrman $(P=0.03)$ and metastasis $(P=0.011)$. Furthermore, Kaplan-Meier survival analysis indicated that ccRCC patients with the higher levels of LncRNA-SARCC had longer overall survival than those with the lower levels of LncRNA-SARCC (Figure 4m; log-rank test, $P<0.01$ ).

Together, results from Figure 4 and Supplementary Figure S4 revealed that LncRNA-SARCC expression in primary $\mathrm{CCRCC}$ tissues was negatively correlated with the endogenous AR, AKT, MMP-13, K-RAS and P-ERK expression, whereby elevated expression of LncRNA-SARCC was associated with better prognosis of cCRCC.

LncRNA-SARCC was regulated by DNA methylation in ccRCC. We next tested the hypothesis that the downregulation of LncRNA-SARCC expression in human cCRCC

\footnotetext{
Figure 5 LncRNA-SARCC was regulated by DNA methylation in ccRCC. (a) MethPrimer software analysis predicted that CpG islands are located at the promoter region of LncRNA-SARCC, which is embedded at chromosome 3 from 2186-2358 (a), 2566-3273 (b), 3345-3899 (c). (b) The DNA methylation levels of LncRNA-SARCC promoter were detected in 20 paired ccRCC tissues and adjacent non-cancer tissues by bisulfite sequencing. The differences in DNA methylation levels between ccRCC tissues and adjacent non-cancer tissues were calculated using Student's $t$-test. (c) Representative sequencing analysis from clinical samples revealed that unmethylated CpG sites in ccRCC tissues were lower than the adjacent normal renal tissues. Hollow and solid circles denoted unmethylated and methylated $\mathrm{CpG}$ sites, respectively. Each row represents a single clone. (d) The association between LncRNA-SARCC expressions and the DNA methylation status of LncRNA-SARCC was evaluated using a Spearman's correlation analysis. (e) The methylation status of the CpG island is assessed before and after 5'-AZA treatment in RCC cell lines (SW839 and OSRC-2) or HK2 cells
} 
tissues and cell lines was caused by an epigenetic mechanism such as DNA methylation. MethPrimer software analysis predicted that $\mathrm{CpG}$-rich islands were located at the promoter region of LncRNA-SARCC, which was embedded at chromosome 3 from 2186-2358(A), 2566-3273(B), 3345-3899(C) (Figure $5 \mathrm{a}$ ). We selected region $\mathrm{A}$ with $13 \mathrm{CpGs}$ to explore a

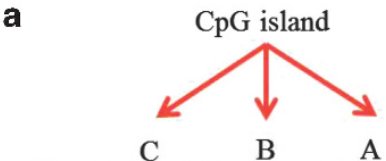

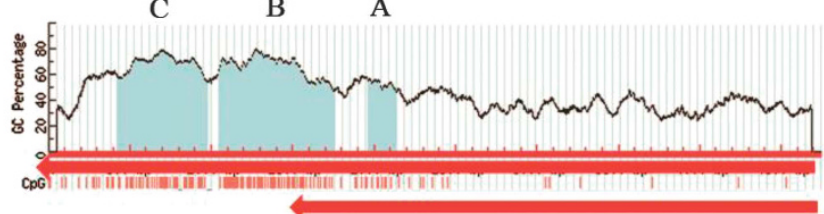

LncRNA-SARCC promoter

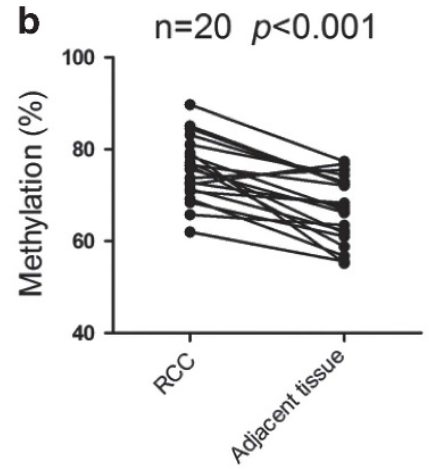

Case 1

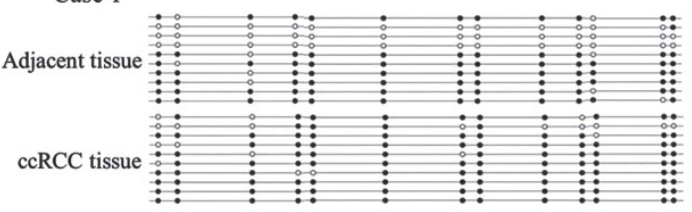

Case 8

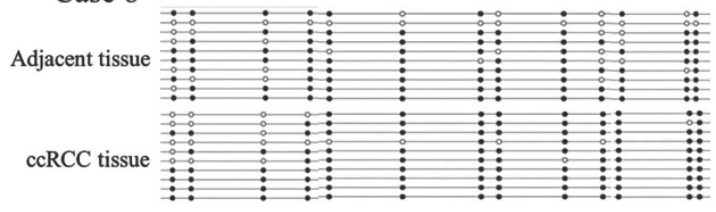

Case 4
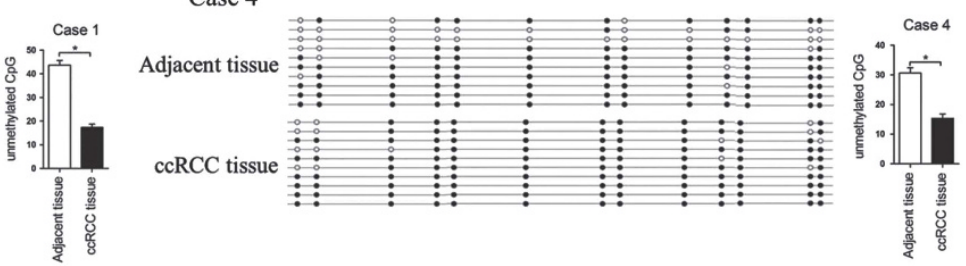

Case 15
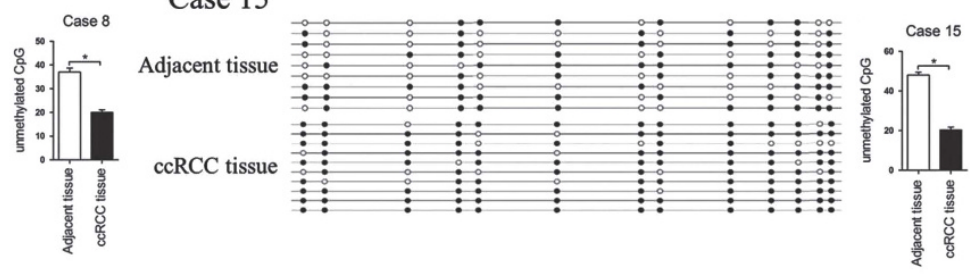

d

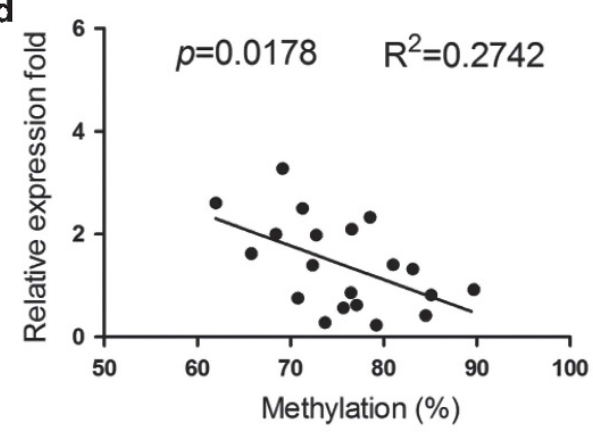

e

HK2

SW839

OSRC-2
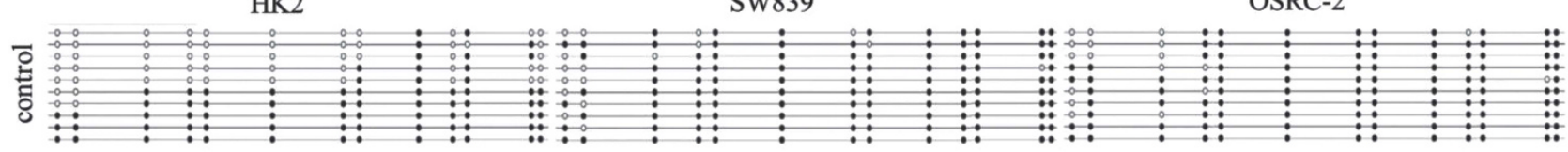

选
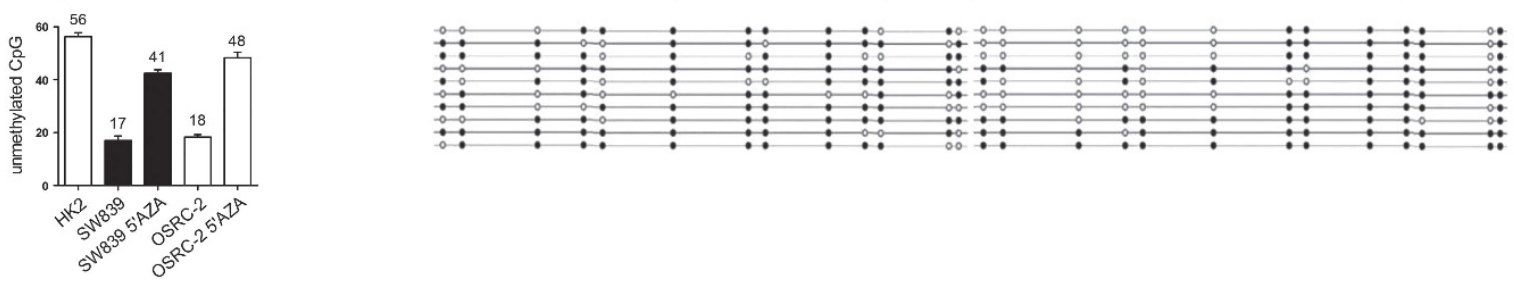
the potential DNA methylation status of LncRNA-SARCC promoter as shown in Supplementary Figure S5A. We measured DNA methylation levels across the LncRNASARCC promoter in 20 pairs of ccRCC tumors and adjacent normal tissues and each of the $13 \mathrm{CpGs}$ was determined by bisulfite analysis. The results illustrated the hyper-methylation of the LncRNA-SARCC promoter in ccRCC tissues compared with adjacent non-tumorous renal tissues (Figure 5b). Furthermore, representative sequencing analysis from clinical samples revealed that unmethylated $\mathrm{CpG}$ sites in ccRCC tissues were lower than in the adjacent normal renal tissues (Figure 5c). Besides, a pronounced negative correlation between LncRNA-SARCC promoter methylation and LncRNA-SARCC expression was observed (Figure 5d). We then examined the methylation status of the same genomic location in SW839, OSRC-2 and HK2 cells. As shown in Figure 5e, the CpG island was highly methylated in SW839 and OSRC-2 cells, but poorly methylated in HK2 cells. The expression of LncRNA-SARCC was determined by qRT-PCR after the cells were treated with vehicle, $5 \mu \mathrm{M}$ and $10 \mu \mathrm{M}$ $5^{\prime}$-azacytidine (5'-AZA). Compared with DMSO, the level of LncRNA-SARCC was dramatically induced in SW839 by almost threefold and fivefold and in OSRC-2 for nearly sevenfold and ninefold in a dose-dependent manner (Supplementary Figures S5B and $\mathrm{S} 5 \mathrm{C}$ ), but reduced in HK2 cells (Supplementary Figure S5D).

Together, results from Figure 5 and Supplementary Figure S5 indicated that LncRNA-SARCC downregulation might be mediated by DNA methylation in cCRCC cells and tissues.

\section{LncRNA-SARCC suppressed RCC cell progression through altering AR in orthotopic xenografts. To further validate that LncRNA-SARCC might serve as a suppressor in vivo, we tested its function in a xenograft tumor model. OSRC-2 cells with firefly luciferase expression were trans- fected with shRNA-SARCC or shRNA-control and the stable clones were inoculated into the left kidney capsule of nude mice and tumor growth and metastasis were evaluated. As shown in Figure 6a, a dramatic induction of luciferase expression in tumors of the shRNA-SARCC group was detected by the IVIS as early as the 4th week, showing promotion of tumor proliferation and metastases compared with the shRNA-control group (Figures $6 b$ and $c$ ). Further- more, shRNA-SARCC facilitated lung, liver, spleen, stomach, right renal and diaphragm metastases (Figure 6d). Con- versely, stable transfection of oe-SARCC into OSRC-2 cells led to markedly reduced growth and tumor weight of orthotopic xenograft tumors (Figure 6e). LncRNA-SARCC}

upregulation decreased metastasis, whereas oe-AR into oe-SARCC cells mostly abolished this decrease (Figures $6 e$ and f).

Together, results from Figure 6 demonstrated that AR-dependent LncRNA-SARCC functioned as a critical tumor suppressor in RCC by suppressing tumorigenesis, local invasion and metastatic colonization.

Sunitinib repressed $\mathrm{RCC}$ progression via inducing LncRNA-SARCC. To test whether the LncRNA-SARCC could be a potential therapeutic target for RCC progression, we examined whether Sunitinib, a tyrosine kinase inhibitor in current clinical use, might be involved with LncRNA-SARCC. Consistent with a previous report, ${ }^{43}$ Sunitinib treatment effectively attenuated RCC cell invasion, migration and proliferation (Supplementary Figures S6A-S6C). In parallel, Sunitinib treatment increased LncRNA-SARCC mRNA and decreased $A R$ protein in a dose-dependent manner in both cells (Figures $7 a$ and $b$ ). A previous study reported that Sunitinib could activate p53 to induce cellular senescence in RCC cells. ${ }^{44}$ Consistent with this, we found p53 protein level could be upregulated by treating SW839 cells with $5 \mu \mathrm{M}$ Sunitinib (Supplementary Figure S6D). Indeed, an examination of LnCRNA-SARCC promoter by PROMO analysis suggested that there might be two p53-binding sites in its $1 \mathrm{~kb}$ promoter region (Supplementary Figure S6E). This mechanistic connection was further substantiated through finding that Sunitinib-enhanced LncRNA-SARCC expression was significantly reduced in cells with a concomitant shRNAmediated $\mathrm{p} 53$ reduction (Figure $7 \mathrm{c}$ ).

To test whether Sunitinib modulated LncRNA-SARCC to impact its therapeutic effect, we prepared RCC cells with and without knocked down LncRNA-SARCC and treated them with $5 \mu \mathrm{M}$ Sunitinib for $48 \mathrm{~h}$. As shown in Figures $7 \mathrm{~d}-\mathrm{g}$, cells with shRNA-SARCC showed more proliferation, migration, invasion as well as less reduction of $A R$ protein compared with the control cells, suggesting that LncRNA-SARCC was a key mediator to influence Sunitinib efficacy. We further confirmed this conclusion in vivo with and without LncRNA-SARCC via treating with Sunitinib as previously described. ${ }^{45,46}$ As shown in the Figure $7 \mathrm{~h}$, shRNA-SARCC blunted the therapeutic efficacy of Sunitinib in RCC xenografts. In a similar manner, Sunitinib treatment repressed local tumor invasion in vivo while depletion of LncRNA-SARCC minimized this effect (Figure 7i).

Together, these data from Figure 7 and Supplementary Figure S6 demonstrated that Sunitinib inhibited RCC tumor progression via altering LncRNA-SARCC expression.

Figure 6 LnCRNA-SARCC suppressed RCC cell progression through altering AR in orthotopic xenografts. (a) Representative images of mice viewed by IVIS Lumina II system in shRNA-SARCC and shRNA-control group 4 weeks after left renal capsule injections $(n=8)$. (b) Macroscopic appearance of the tumor xenografts in nude mice from the 7- to 8-week group with OSRC-2 cells with or without shRNA-SARCC (upper). Weights of the xenografts were also shown (mean \pm S.D.) $(n=8)$ (lower). (c) Presented are representative images of abdominal metastasis viewed by IVIS Lumina II system in each group 4 weeks after the orthotopic xenograft transplantation ( $n=8$ ). (d) Macroscopic appearance and representative images of metastatic organs viewed by IVIS in shRNA-SARCC and shRNA-control transfected OSRC-2 cells 4 weeks after left renal capsule injections $(n=8)$. Quantitations shown in lower panel. (e) Macroscopic appearance of the tumor xenograft in nude mice from the 8- to 9-week group with OSRC-2 xenografts (upper). Weights of the xenografts were shown (mean \pm S.D.) $(n=8)$ (lower). (f) Orthotopic xenograft animal models were also generated using mock, oe-AR, oeRNA-SARCC and oeRNA-SARCC+ oe-AR transfected OSRC-2 cells. Presented are representative images (left) of abdominal metastasis viewed by IVIS in each group 4 weeks after the orthotopic xenograft transplantation $(n=8)$. Quantitations shown at right. $(\mathbf{g})$ A schematic illustration of the proposed model depicting LncRNA-SARCC inhibits the invasion, migration and proliferation of renal cell carcinoma cells through modulating AR/miR-143-3p signals. Data shown are mean \pm S.D. $(n=8)$. For $\mathbf{a}, \mathbf{c}, \mathbf{d}, \mathbf{e}, \mathbf{h}$, and $\mathbf{i}$, ${ }^{*} P<0.05$ 
a

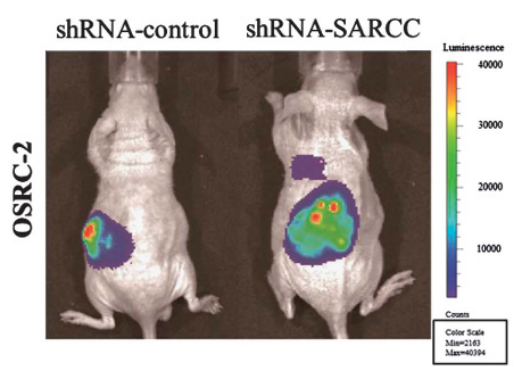

d

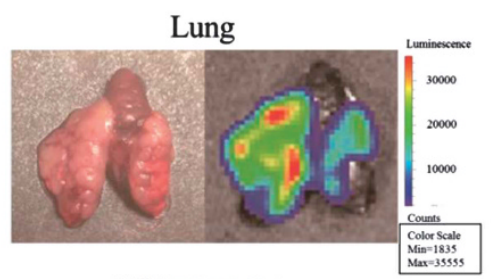

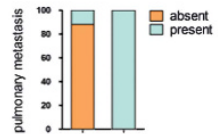

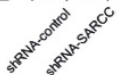

Right Kidney
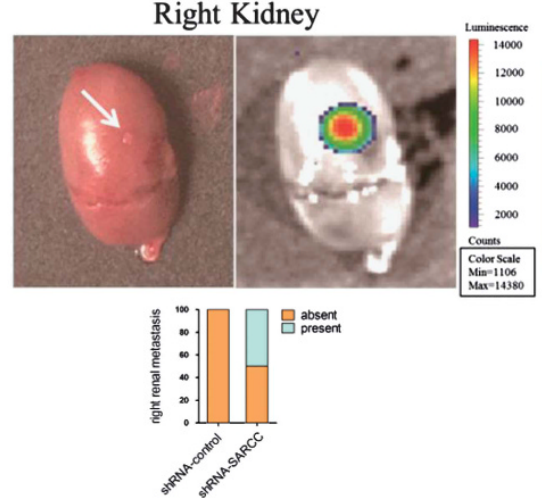

e
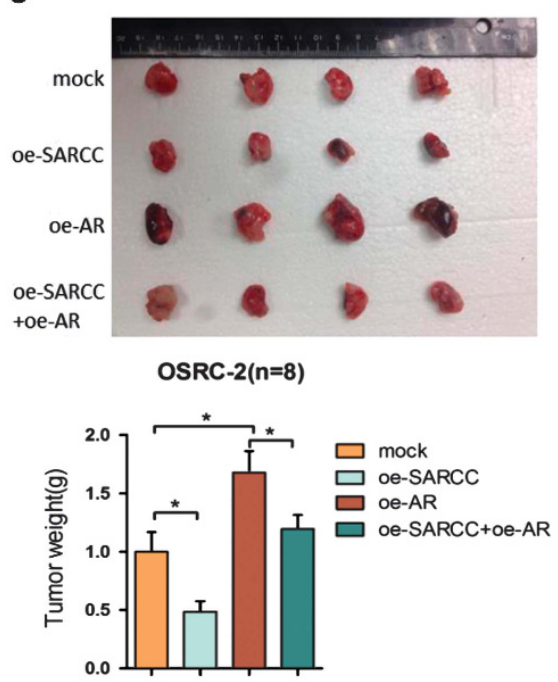

b
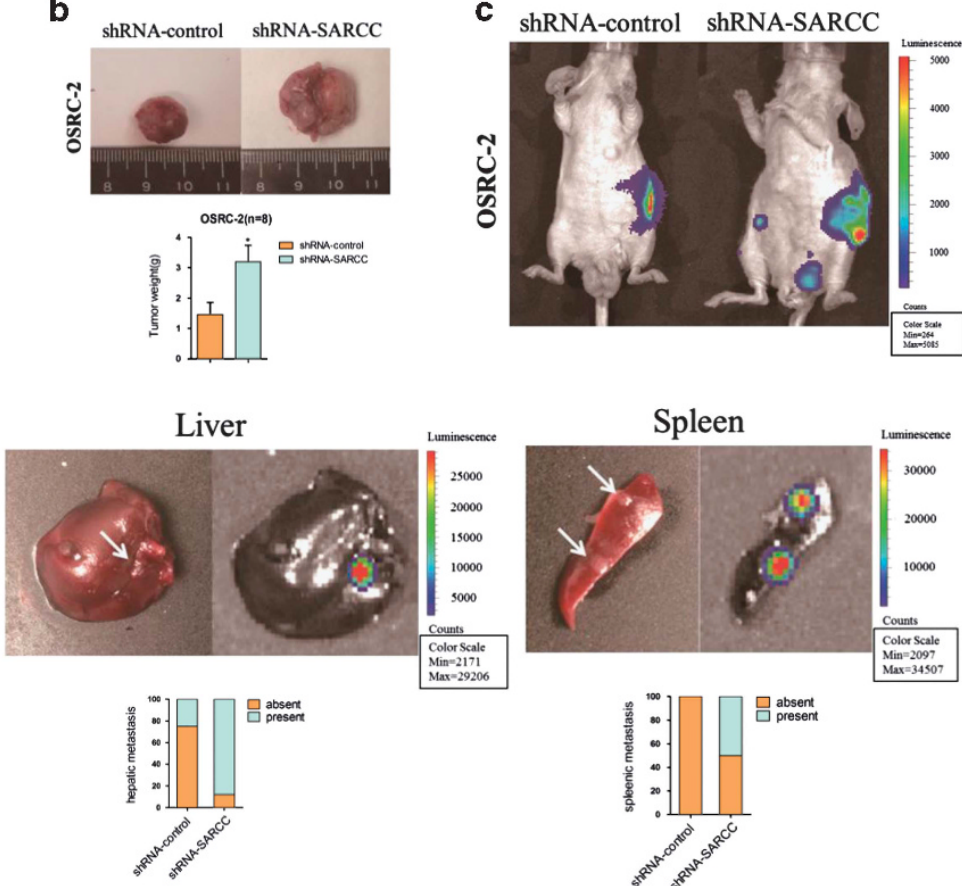

Stomach

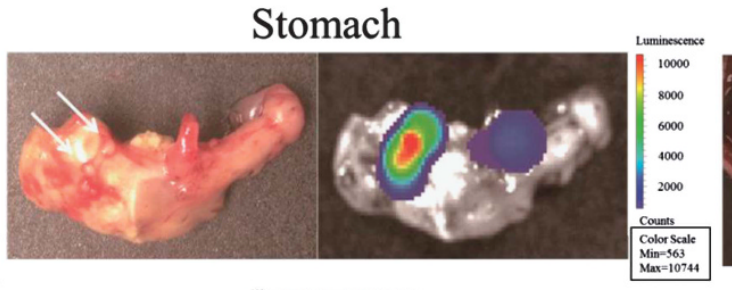

Diaphragm
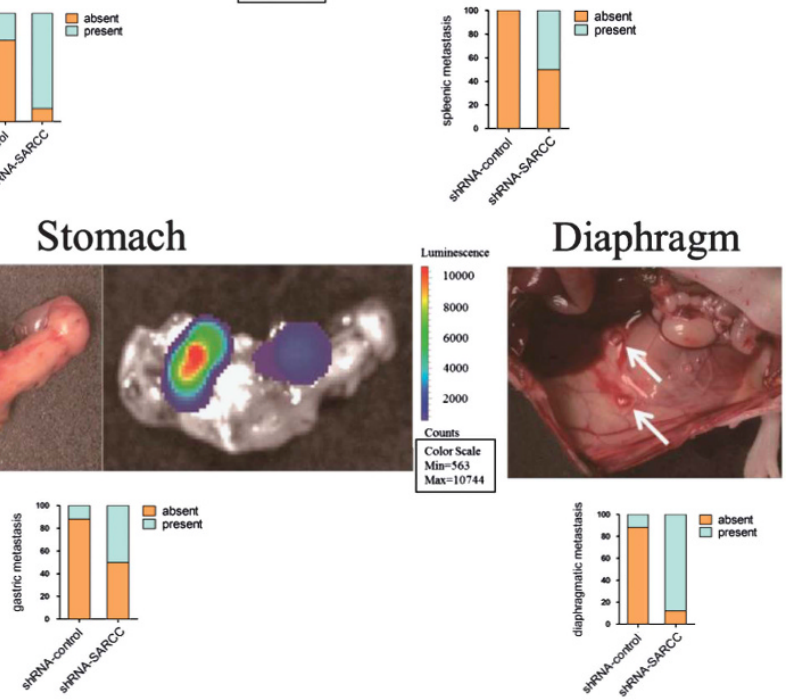

f

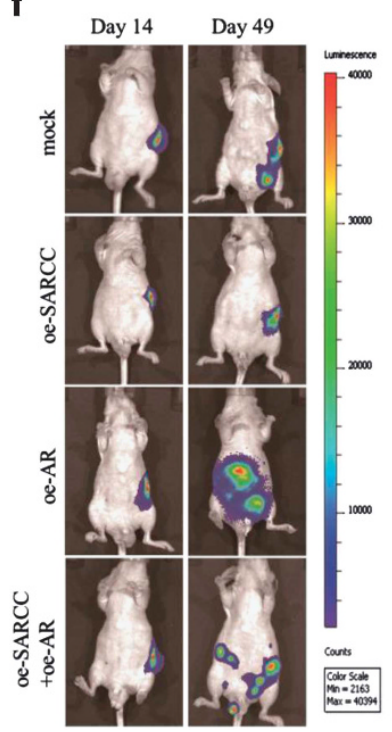

g

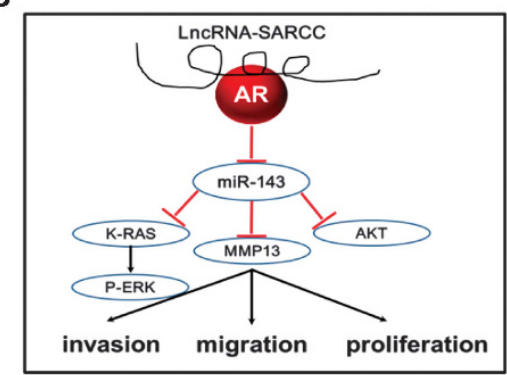

OSRC-2(n=8)

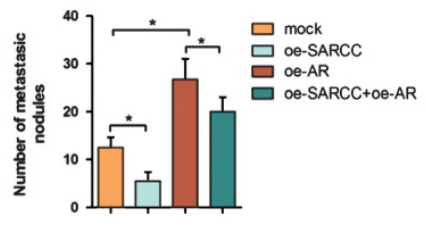




\section{Discussion}

In this study, we reported that LncRNA-SARCC functioned as a tumor suppressor in RCC through repressing AR activity by physical interaction. Expression of LncRNA-SARCC in cCRCC and metastatic CCRCC was reduced compared with that in surrounding non-tumor and non-metastatic tissues with a positive correlation ccRCC prognosis. These lower LncRNASARCC levels were associated with enhanced ccRCC invasion, migration, proliferation and resistance to Sunitinib therapy, via its regulation of AR that suppressed miR-143-3p expression to influence downstream genes such as K-RAS, MMP-13, AKT and P-ERK expression.

Early studies have suggested that AR acts as an oncogenic protein to promote RCC cells initiation and progression. ${ }^{6}$ Our report further substantiated the significance of AR in RCC by demonstrating that an interacting molecule such as LncRNASARCC functioning through AR could regulate RCC invasion, migration and proliferation both in vitro and in vivo. On the other hand, it has also been reported that AR expression is low in selected RCC tumors and cell lines, raising the issue whether AR functionally promotes the RCC development. ${ }^{47}$ It is possible that AR's role in RCC progression is stagedependent, and its expression dictates a differential fate of the RCC metastasis and progression. Interestingly, LncRNASARCC was found to be overexpressed in six RCC tissues in a LncRNA microarray survey, ${ }^{23}$ contradicting our conclusion that LncRNA-SARCC expression is lower in RCC than in adjacent non-cancerous tissues. Several reasons might explain this potential difference. One is that the result from a microarray of a limited sample number can be reversed upon further testing with more samples. Second, a different platform of testing, that is, microarray versus qPCR might lead to different outcomes. Third, LncRNA-SARCC expression may be tightly linked with the tissue specimen preparation in that surgery-induced ischemia might differentially regulate its expression in normal and tumor tissues. Fourth, it is also possible that LncRNASARCC expression in RCC is stage-dependent like AR, which may be critical for late stage RCC development.

Some reports of miRNA signatures showed that miR-143$3 p$ might function as a tumor suppressor and is frequently down-regulated in several types of cancers, including RCC. ${ }^{34,48-50}$ Our studies supported these findings as AR could directly bind to the miR-143-3p promoter and thus transcriptionally suppress miR-143-3p expression, whereas LncRNA-SARCC could post-transcriptionally inhibit AR protein, thus increasing miR-143-3p expression.
Significantly, we find that Sunitinib induces LncRNASARCC expression, and the level of LncRNA-SARCC determines the sensitivity to this drug. Therefore, Sunitinib efficacy is potentially linked with LncRNA-SARCC, which serves as a useful biomarker for drug effectiveness and is used to segregate patients into different responsive populations to save both money and time for the non-responsive patients. This finding also points to a novel therapeutic approach to enhance Sunitinib efficacy in RCC treatment through enhancing the expression of LncRNA-SARCC.

In summary, our report demonstrates that LncRNA-SARCC functions as a tumor suppressor to control RCC development through regulating $\mathrm{AR} / \mathrm{miR}-143-3 p$ signals. Targeting this newly identified signal pathway may help us to better suppress RCC progression.

\section{Materials and Methods}

Human samples. Surgical specimens from human ccRCC tissues were obtained from 66 patients and 8 metastatic ccRCC patients in the Department of Urology, Shanghai Tenth People's Hospital, Tongji Medical School (Shanghai, China), freshly frozen in liquid nitrogen and stored at $-80^{\circ} \mathrm{C}$ until use. Informed consent was obtained from patients and the study was approved by the Institutional Review Board of Tongji Medical College.

Immunohistochemistry. Immunohistochemical (IHC) staining was performed as previously described, ${ }^{6,51}$ with antibodies specific for AKT, P-ERK, AR, Hsp90 (Abcam Inc., Cambridge, MA, USA; 1:200 dilution) and MMP-13 and K-RAS (Santa Cruz Biotechnology, Santa Cruz, CA, USA; 1:200 dilution). The reactivity degree was assessed by at least two pathologists without knowledge of the clinicopathological features of the tumors. The degree of positivity was initially classified according to scoring both the proportion of positive staining tumor cells and the staining intensities. Scores representing the proportion of positively stained tumor cells were graded as: $0(<10 \%) ; 1(11-25 \%) ; 2$ (26-50\%); $3(51-75 \%)$ and $4(>75 \%)$. The intensity of staining was determined as: 0 (no staining); 1 (weak staining =light yellow); 2 (moderate staining=yellow brown); and 3 (strong staining $=$ brown). The staining index was calculated as the product of staining intensity $\times$ percentage of positive tumor cells, resulting in scores of $0,1,2,3,4,6,8$, 9 and 12 . Only cells with clear tumor cell morphology were scored.

Fluorescent in situ hybridization. FISH was performed as previously described. ${ }^{16}$ It is a powerful technique that uses non-toxic fluorescent DNA probes to target any given sequence within a nucleus, resulting in colored signals that are detected with a fluorescence microscope and was performed at Biosense Co. Ltd (Guangzhou, China). Paraffin-embedded tissue blocks were retrospectively retrieved from renal cancer, colon cancer, gastric cancer, prostate cancer, bladder cancer and liver cancer patients. Quantum dot FISH was performed to detect the presence of LnCRNA-SARCC using a digoxin-labeled oligonucleotide probe (Supplementary Table 3) indirectly labeled with digoxin-antibody-conjugated quantum dots.

Cell culture. The human RCC cell lines, SW839, OSRC-2, A498, 769-P, 786-0, Caki-1, Caki-2 and the immortalized proximal tubule epithelial cell line from normal adult human kidney, HK2, were originally purchased from American Type Culture

Figure 7 Sunitinib repressed RCC progression via inducing LncRNA-SARCC. (a and $\mathbf{b})$ qRT-PCR assays for LncRNA-SARCC expression (a) and western blot analysis for AR protein levels (b) with 2.5 and $5 \mu \mathrm{M}$ Sunitinib treatment in SW839 and OSRC-2 cells. (c) Western blot analysis for p53 protein levels with shRNA-p53 in SW839 cells (left); LncRNA-SARCC expression was measured by Real-time PCR assays in SW839 cells expressing shRNA-p53 or treated with $5 \mu$ M Suni and shRNA-p53 $+5 \mu M$ Suni (right). (d-f) Representative images (left) and number of invasive cells per high-power field (right) (d), representative micrographs of wound-healing assay and number of the indicated cells (right) (e) and MTT proliferation change (f) was induced by transfection of shRNA-SARCC in SW839 (left) and OSRC-2 cells (right) versus shRNA-control cells after treatment with $5 \mu \mathrm{M}$ Sunitinib. Wound closures were photographed at 0 and $24 \mathrm{~h}$ after wounding. (g) Western blot analysis for AR protein levels with shRNA-SARCC compared with shRNA-control in SW839 and OSRC-2 cells after treatment with $5 \mu \mathrm{M}$ Sunitinib. (h) Macroscopic appearance of tumor xenografts in nude mice of the 8- to 9-week group of mice xenografted with OSRC-2 cells (upper). Weights of the xenografts were also shown $(n=8)$ (lower). (i) Orthotopic xenograft animal models were also generated using shRNA-SARCC or shRNA-control in OSRC-2 cells and mice treated with $5 \mu \mathrm{M}$ Sunitinib. Presented are representative images (left) of abdominal metastasis viewed by IVIS Lumina II system in each group 4 weeks after the orthotopic xenograft transplantation $(n=8)$. Quantitation shown at right. Data shown $\operatorname{are}$ mean \pm S.D. $(n=8)$. ${ }^{*} P<0.05$ 


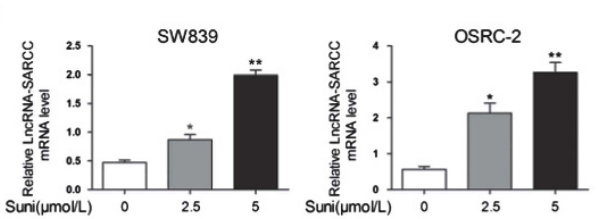

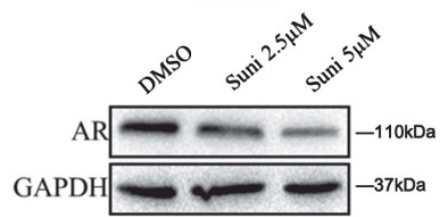

OSRC-2

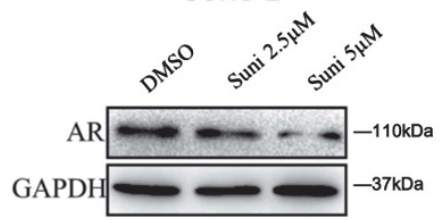

C

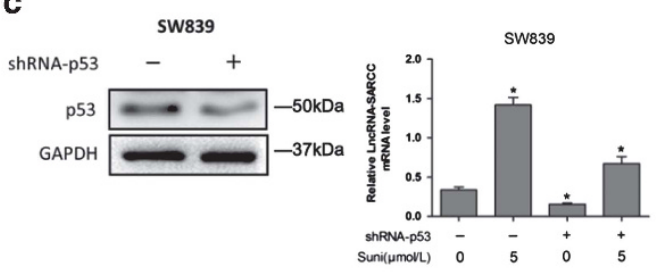

d

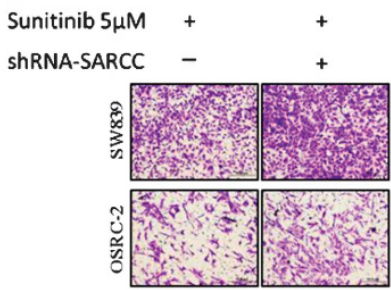

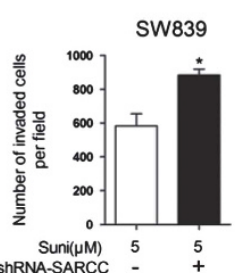

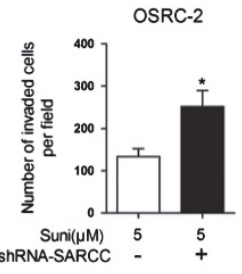

e
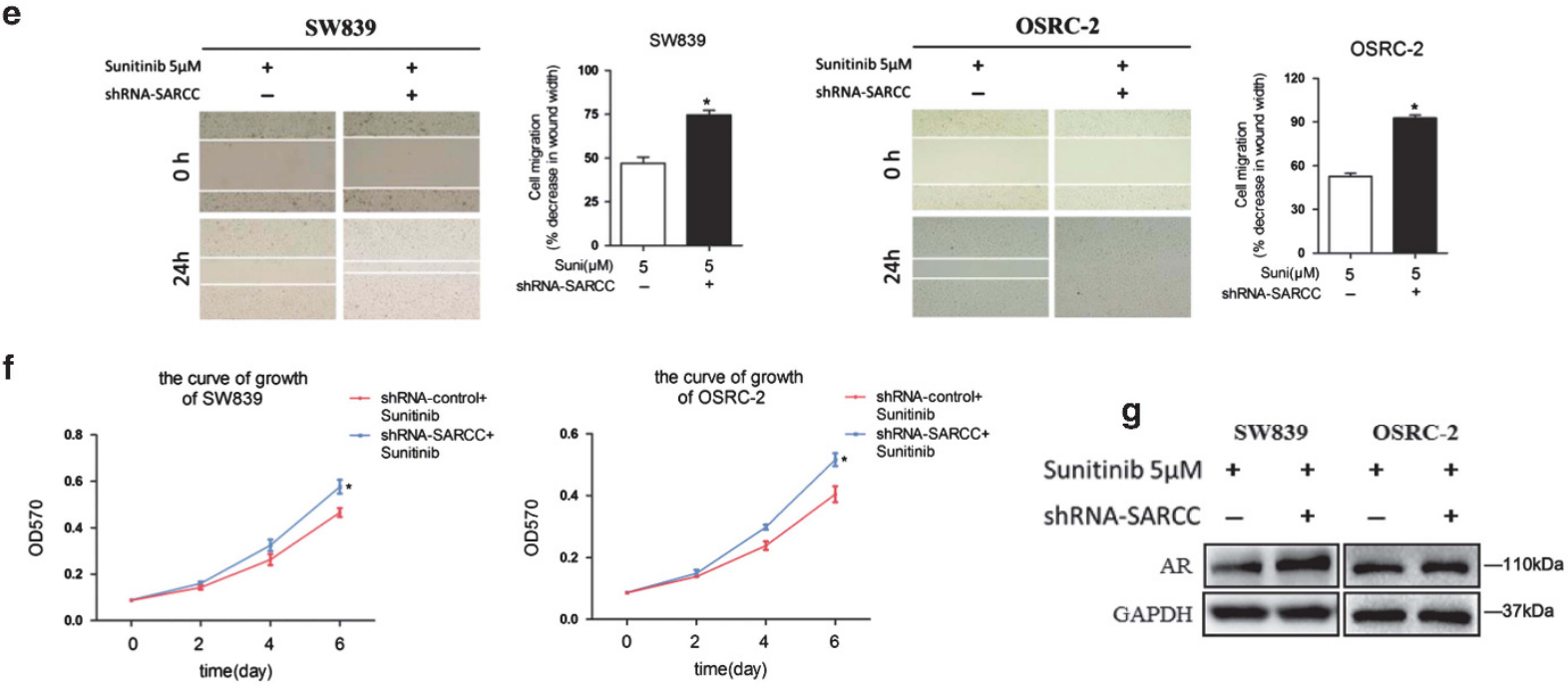

h

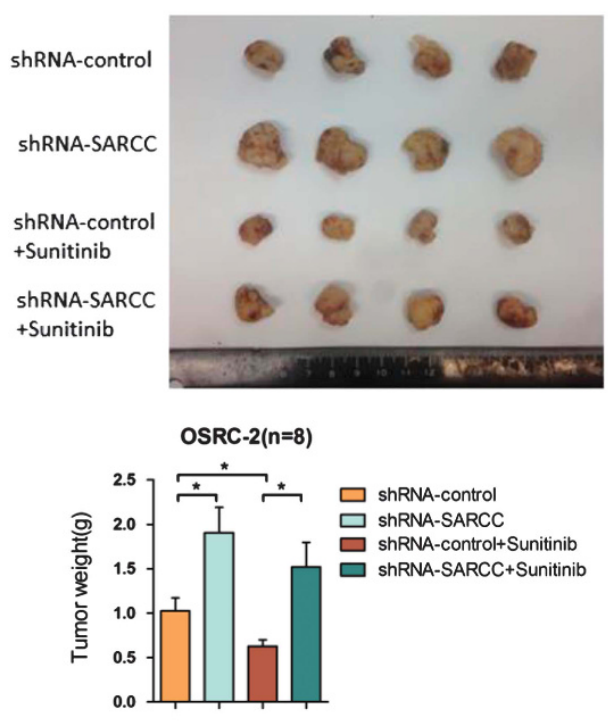

i

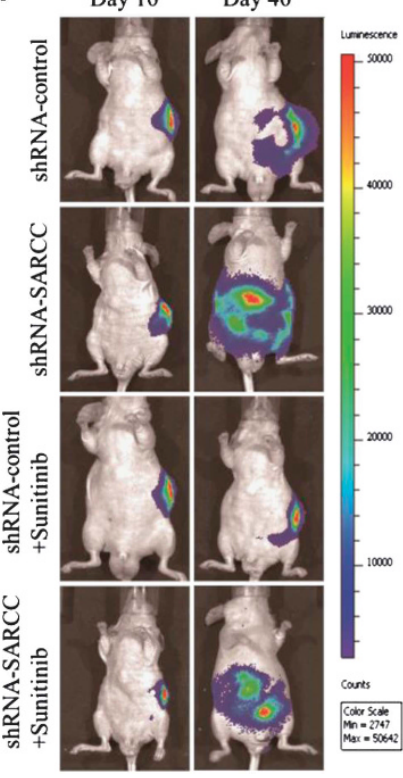

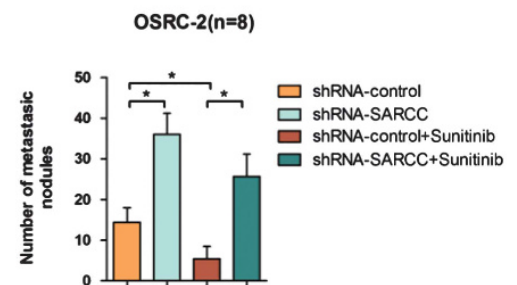


Collection (ATCC, Manassas, VA, USA) and preserved in our laboratory as ampules in Liquid N2. A new ampule was reconstituted for use in experiments approximately every 3 months as needed. All RCC cells were cultured in DMEM (Invitrogen, Carlsbad, CA, USA) supplemented with $10 \%$ fetal bovine serum (FBS) in the humidified $5 \% \mathrm{CO}_{2}$ environment at $37^{\circ} \mathrm{C}$.

RNA immunoprecipitation. Native RIP was performed as described previously. $^{52}$ In brief, SW839 or OSRC-2 cells were lysed in RIPA lysis buffer (20 mM Tris-HCl/pH 7.5, 150 mM NaCl, 1 mM Na 2 EDTA, 1 mM EGTA, 1\% NP-40, $1 \%$ sodium deoxycholate, $2.0 \mathrm{mM}$ sodium pyrophosphate, $1 \mathrm{mM}$ beta-glycerophosphate, $1 \mathrm{mM} \mathrm{Na}_{3} \mathrm{VO}_{4}$ and $1 \mu \mathrm{g} / \mathrm{ml}$ leupeptin) supplemented with anti-RNase, protease inhibitor cocktail. RNase-free DNase (NEB) (400U) was added to the lysate, incubated on ice for $30 \mathrm{~min}$, diluted in the RIPA buffer and $50 \mu$ l supernatant was saved as input for PCR analysis. The $500 \mu \mathrm{l}$ supernatant was incubated with $4 \mu \mathrm{g}$ AR antibody (normal rabbit IgG overnight as control). Protein $A / G$ beads were pre-blocked by $15 \mathrm{mg} / \mathrm{ml} \mathrm{BSA}$ in PBS, then added to the antibody-lysate mixture, incubated for another $2 \mathrm{~h}$ and the washed four times by RIPA buffer supplemented with anti-RNase, protease inhibitor cocktail. The RNA was extracted using Trizol (Invitrogen) according to the manufacturer's protocol and subjected to RT-qPCR analysis. For UV cross-linking and RIP, cells were first subjected to UV cross-linking $\left(200 \mathrm{~mJ} / \mathrm{cm}^{2}\right)$ and then conducted as native RIP protocol.

RNA pull-down assay. RNA pull-down assay was performed as described. ${ }^{52}$ In brief, T7 promoter tagged oligos were produced by PCR. Biotin-labeled LncRNASARCC RNA (GFP RNA as control) was in vitro transcribed with the Biotin RNA labeling mix (Roche, Branford, CT, USA) and T7 RNA polymerase (Roche) and purified with RNeasy MiniKit (Qiagen, Germantown, MD, USA). RNAs were incubated with nuclear extract from SW839 cells in the presence of anti-RNase, protease inhibitor cocktail. Proteins pulled down by biotinylated RNA were subjected to western blot analysis.

Chromatin immunoprecipitation assay (ChIP). Cells were crosslinked with $4 \%$ formaldehyde for 10 min followed by cell collection and sonication with a predetermined power to yield genomic DNA fragments of 300-1000 bp long. Lysates were precleared sequentially with normal rabbit IgG (sc-2027, Santa Cruz Biotechnology) and protein A agarose. Anti-AR antibody $(2.0 \mu \mathrm{g})$ was added to the cell lysates and incubated at $4{ }^{\circ} \mathrm{C}$ overnight. For the negative control, IgG was used in the reaction. Specific primer sets designed to amplify a target sequence within human miR-143-3p promoter were listed in Supplementary Table 2; PCR products were analyzed by agarose gel electrophoresis.

RCC cell proliferation assay. RCC cells were seeded in 24-well plates (3000 cells/well) and cultured for $24,48,72$ or $96 \mathrm{~h}$. Cells were harvested and cell numbers were calculated using MTT agent. DMSO was used as the control. We added $250 \mu \mathrm{l}$ of $5 \mathrm{mg} / \mathrm{ml} \mathrm{MTT}$ to each well, incubated for $2 \mathrm{~h}$ in incubator at $37^{\circ} \mathrm{C}$, removed the media and added $150 \mu \mathrm{l}$ DMSO. We then covered with tinfoil and agitated cells on orbital shaker for $15 \mathrm{~min}$ and then read the absorbance at $570 \mathrm{~nm}$.

Wound-healing assay. Indicated cells were plated to confluence in six-well plates. Streaks across the plate were created in the monolayer with a pipette tip. Progression of migration was observed and photographed at $24 \mathrm{~h}$ after wounding. The data shown were representative micrographs of wound-healing assay of the indicated cells. Wound closures were photographed at 0 and $24 \mathrm{~h}$ after wounding. Experiments were repeated at least three times with similar results, and error bars represented mean \pm S.D. ${ }^{*} P<0.05$.

RCC cell invasion assay. The invasive capability of RCC cells was determined by the Transwell assay. RCC cells were harvested and seeded with serum-free DMEM into the upper chambers at $5 \times 10^{4}$ cells/well, and the bottom chambers contained DMEM with $10 \%$ FBS, and then transwells incubated for $24 \mathrm{~h}$ at $37^{\circ} \mathrm{C}$. Following incubation, the invasive cells attached to the lower surface of the membrane were fixed by $4 \%$ paraformaldehyde and stained with $1 \%$ toluidine blue. Cell numbers were counted in five randomly chosen microscopic fields $(\times 100)$ per membrane.

Luciferase assay. Cells were plated in 24-well plates and transfected with ARE-luc pGL3 using Lipofectamine (Invitrogen) according to the manufacturer's instruction. After transfection, DMEM media containing charcoal-stripped FBS was added into the culture with addition of various concentrations of DHT with ethanol as vehicle control. pRL-TK was used as internal control. Luciferase activity was measured by Dual-Luciferase Assay (Promega, Madison, WI, USA) according to the manufacturer's manual.

Bisulfite sequencing PCR. DNA methylation analysis was performed as previously described. ${ }^{53}$ In brief, genomic DNA was extracted from RCC tissues specimens according to the Kit instructions (Shanghai Integrated Biotech Solution Co. Ltd., Shanghai, China). The SW839 and OSRC-2 cell lines were treated with 5Aza-CdR $(1 \mu \mathrm{M})$ for $72 \mathrm{~h}$ while HK2 cells were treated with $0.1 \%$ DMSO. Genomic DNA was extracted using the cell/tissue genomic DNA extraction kit instructions according to the manufacturer's instructions. The bisulfite treatment was performed according to DNA Methylation Kit protocol (Qiagen, cat\# 59824). The bisulfate primers used in this study were designed using MethPrimer. The methylation of the sample was analyzed using BiQ Analyzer software.

Mouse model of orthotopic tumor implantation. OSRC-2 cells transfected with pLKO1-shRNA-SARCC, pLKO1-shRNA-control, pLKO1-shRNASARCC+ Sunitinib, pLKO1-shRNA-control+ Sunitinib (for knock down experiments), pWPI-mock, pWPI-oe-SARCC, pWPI-oe-AR or pWPI-oe-SARCC+ pWPI-oe-AR (for overexpression experiments), were injected at $2 \times 10^{6}$ cells/mouse, into the left subrenal capsule of 6 -week-old male athymic nude mice (from NCl) ( $n=8$ mice/group). Cells were also transduced with luciferase for the non-invasive in vivo imaging system that was performed once a week. We also performed experiments with additional groups (OSRC-2 cells with/without shRNA-SARCC) of mice treated with/ without Sunitinib (orally, once a day, at the dose levels of $40 \mathrm{mg} / \mathrm{kg}$ body weight). After 8-9 weeks, mice were killed, and tumors were excised and weighed. Studies on animals were conducted with approval from the Animal Research Ethics Committee of the University of Rochester Medical Center.

Statistical analysis. Unless otherwise stated, all data were shown as mean \pm S.D. The SPSS 12.0 statistical software (SPSS Inc., Chicago, IL, USA) was applied for statistical analysis. $P<0.05$ was regarded as the threshold value for statistical significance.

\section{Conflict of Interest}

The authors declare no conflict of interest.

Acknowledgements. This work was sponsored by NIH grants (CA155477 and CA156700), the National Science Foundation of China (31570775 and 81602216), National Science Foundation of Shanghai (16ZR1426500), Shanghai Pujiang Program (16PJD037) and Distinguished Young Scholars of Tongji University (2014KJ094), George Whipple Professorship Endowment and Taiwan Department of Health Clinical Trial, Research Center of Excellence (DOH99-TD-B-111-004 to China Medical University, Taichung, Taiwan). We are grateful to Liang Xu of Shanghai Integrated Biotech Solution Co. Ltd. for technical assistance.

1. Mulders PF, Brouwers AH, Hulsbergen-van der Kaa CA, van Lin EN, Osanto S, de Mulder PH. [Guideline 'Renal cell carcinoma']. Ned Tijdschr Geneeskd 2008; 152: 376-380.

2. Ramana J. RCDB: renal cancer gene database. BMC Res Notes 2012; 5: 246.

3. Najjar YG, Rini BI. Novel agents in renal carcinoma: a reality check. Ther Adv Med Oncol 2012; 4: 183-194

4. Cho IC, Chung J. Current status of targeted therapy for advanced renal cell carcinoma. Korean J Urol 2012; 53: 217-228.

5. Staehler M, Rohrmann K, Haseke N, Stief CG, Siebels M. Targeted agents for the treatment of advanced renal cell carcinoma. Curr Drug Targets 2005; 6: 835-846.

6. He D, Li L, Zhu G, Liang L, Guan Z, Chang L et al. ASC-J9 suppresses renal cell carcinoma progression by targeting an androgen receptor-dependent HIF2alpha/VEGF signaling pathway. Cancer Res 2014; 74: 4420-4430.

7. Orth SR. Cigarette smoking: an important renal risk factor - far beyond carcinogenesis. Tob Induc Dis 2002; 1: 137-155.

8. Feldman DR, Motzer RJ. Novel targets and therapies for metastatic renal cell carcinoma. Oncology (Williston Park) 2006; 20: 1745-1753; discussion 1756.

9. Perkel JM. Visiting "noncodarnia". BioTechniques 2013; 54: 301, 303-304.

10. Cabianca DS, Casa V, Bodega B, Xynos A, Ginelli E, Tanaka $Y$ et al. A long ncRNA links copy number variation to a polycomb/trithorax epigenetic switch in FSHD muscular dystrophy. Cell 2012; 149: 819-831.

11. Prensner JR, Chinnaiyan AM. The emergence of IncRNAs in cancer biology. Cancer Discov 2011: 1: 391-407. 
12. Wang KC, Yang YW, Liu B, Sanyal A, Corces-Zimmerman R, Chen $Y$ et al. A long noncoding RNA maintains active chromatin to coordinate homeotic gene expression. Nature 2011; 472: 120-124.

13. Schmitt AM, Chang HY. Long noncoding RNAs in cancer pathways. Cancer Cell 2016; 29: 452-463.

14. Qi P, Zhou XY, Du X. Circulating long non-coding RNAs in cancer: current status and future perspectives. Mol Cancer 2016; 15: 39.

15. Wang Kevin C, Chang Howard Y. Molecular mechanisms of long noncoding RNAs. Mol Cell 2011; 43: 904-914.

16. Yuan JH, Yang F, Wang F, Ma JZ, Guo YJ, Tao QF et al. A long noncoding RNA activated by TGF-beta promotes the invasion-metastasis cascade in hepatocellular carcinoma. Cancer Cell 2014; 25: 666-681.

17. Gupta RA, Shah N, Wang KC, Kim J, Horlings HM, Wong DJ et al. Long non-coding RNA HOTAIR reprograms chromatin state to promote cancer metastasis. Nature 2010; 464: 1071-1076.

18. Mehra R, Shi Y, Udager AM, Prensner JR, Sahu A, lyer MK et al. A novel RNA in situ hybridization assay for the long noncoding RNA SChLAP1 predicts poor clinical outcome after radical prostatectomy in clinically localized prostate cancer. Neoplasia 2014; 16 : 1121-1127.

19. Chen $Y$, Sun $Y$, Rao Q, Xu H, Li L, Chang C. Androgen receptor (AR) suppresses miRNA-145 to promote renal cell carcinoma (RCC) progression independent of VHL status. Oncotarget 2015; 6: 31203-31215.

20. Shang D, Yang H, Xu Y, Yao Q, Zhou W, Shi X et al. A global view of network of IncRNAs and their binding proteins. Mol Biosyst 2015; 11: 656-663.

21. Wang KC, Chang HY. Molecular mechanisms of long noncoding RNAs. Mol Cell 2011; 43: 904-914.

22. Wright MW, Bruford EA. Naming 'junk': human non-protein coding RNA (ncRNA) gene nomenclature. Hum Genomics 2011; 5: 90-98.

23. Yu G, Yao W, Wang J, Ma X, Xiao W, Li H et al. LncRNAs expression signatures of renal clear cell carcinoma revealed by microarray. PloS One 2012; 7: e42377.

24. Volders PJ, Verheggen K, Menschaert G, Vandepoele K, Martens L, Vandesompele J et al. An update on LNCipedia: a database for annotated human IncRNA sequences. Nucleic Acids Res 2015; 43: 4363-4364.

25. Darouiche RO, Wall MJ Jr, Itani KM, Otterson MF, Webb AL et al. Chlorhexidine-alcohol versus povidone-iodine for surgical-site antisepsis. N Engl J Med 2010; 362: 18-26.

26. Ai J, Wang Y, Dar JA, Liu J, Liu L, Nelson JB et al. HDAC6 regulates androgen receptor hypersensitivity and nuclear localization via modulating Hsp90 acetylation in castrationresistant prostate cancer. Mol Endocrinol 2009; 23: 1963-1972.

27. Basak S, Pookot D, Noonan EJ, Dahiya R. Genistein down-regulates androgen receptor by modulating HDAC6-Hsp90 chaperone function. Mol Cancer Ther 2008; 7: 3195-3202.

28. Byers LA, Diao L, Wang J, Saintigny P, Girard L, Peyton M et al. An epithelial-mesenchymal transition gene signature predicts resistance to EGFR and PI3K inhibitors and identifies AxI as a therapeutic target for overcoming EGFR inhibitor resistance. Clin Cancer Res 2013; 19: 279-290.

29. Schaefer A, Stephan C, Busch J, Yousef GM, Jung K. Diagnostic, prognostic and therapeutic implications of microRNAs in urologic tumors. Nat Rev Urol 2010; 7: 286-297.

30. Hwang HW, Mendell JT. MicroRNAs in cell proliferation, cell death, and tumorigenesis. Br J Cancer 2007; 96: R40-R44.

31. Catto JW, Alcaraz A, Bjartell AS, De Vere White R, Evans CP, Fussel $S$ et al. MicroRNA in prostate, bladder, and kidney cancer: a systematic review. Eur Urol 2011; 59: 671-681.

32. Ribas J, Ni X, Haffner M, Wentzel EA, Salmasi AH, Chowdhury WH et al. miR-21: an androgen receptor-regulated microRNA that promotes hormone-dependent and hormoneindependent prostate cancer growth. Cancer Res 2009; 69: 7165-7169.
33. Kitade $Y$, Akao $Y$. MicroRNAs and their therapeutic potential for human diseases: microRNAs, miR-143 and -145, function as anti-oncomirs and the application of chemically modified miR-143 as an anti-cancer drug. J Pharmacol Sci 2010; 114: 276-280.

34. Kent OA, Chivukula RR, Mullendore M, Wentzel EA, Feldmann G, Lee KH et al. Repression of the miR-143/145 cluster by oncogenic Ras initiates a tumor-promoting feed-forward pathway. Genes Dev 2010; 24: 2754-2759.

35. Hu Y, Ou Y, Wu K, Chen Y, Sun W. miR-143 inhibits the metastasis of pancreatic cancer and an associated signaling pathway. Tumour Biol 2012; 33: 1863-1870.

36. Osaki M, Takeshita F, Sugimoto Y, Kosaka N, Yamamoto Y, Yoshioka Y et al. MicroRNA-143 regulates human osteosarcoma metastasis by regulating matrix metalloprotease-13 expression. Mol Ther 2011; 19: 1123-1130.

37. Wu D, Huang P, Wang L, Zhou Y, Pan H, Qu P. MicroRNA-143 inhibits cell migration and invasion by targeting matrix metalloproteinase 13 in prostate cancer. Mol Med Rep 2013; 8: 626-630.

38. Noguchi S, Mori T, Hoshino Y, Maruo K, Yamada N, Kitade Y et al. MicroRNA-143 functions as a tumor suppressor in human bladder cancer T24 cells. Cancer Lett 2011; 307: 211-220.

39. Higgins JP, Shinghal R, Gill H, Reese JH, Terris M, Cohen RJ et al. Gene expression patterns in renal cell carcinoma assessed by complementary DNA microarray. Am J Patho 2003; 162: 925-932.

40. Yusenko MV, Kuiper RP, Boethe T, Ljungberg B, van Kessel AG, Kovacs G. High-resolution DNA copy number and gene expression analyses distinguish chromophobe renal cell carcinomas and renal oncocytomas. BMC Cancer 2009; 9: 152.

41. Jones J, Otu H, Spentzos D, Kolia S, Inan M, Beecken WD et al. Gene signatures of progression and metastasis in renal cell cancer. Clin Cancer Res 2005; 11: 5730-5739.

42. Gumz ML, Zou H, Kreinest PA, Childs AC, Belmonte LS, LeGrand SN et al. Secreted frizzledrelated protein 1 loss contributes to tumor phenotype of clear cell renal cell carcinoma. Clin Cancer Res 2007; 13: 4740-4749.

43. Huang D, Ding Y, Li Y, Luo WM, Zhang ZF, Snider J et al. Sunitinib acts primarily on tumor endothelium rather than tumor cells to inhibit the growth of renal cell carcinoma. Cancer Res 2010; 70: 1053-1062.

44. Zhu Y, Xu L, Zhang J, Hu X, Liu Y, Yin $\mathrm{H}$ et al. Sunitinib induces cellular senescence via p53/ Dec1 activation in renal cell carcinoma cells. Cancer Sci 2013; 104: 1052-1061.

45. Zhang L, Smith KM, Chong AL, Stempak D, Yeger H, Marrano P et al. In vivo antitumor and antimetastatic activity of sunitinib in preclinical neuroblastoma mouse model. Neoplasia 2009; 11: 426-435.

46. Fuereder T, Jaeger-Lansky A, Hoeflmayer D, Preusser M, Strommer S, Cejka D et al. mTOR inhibition by everolimus counteracts VEGF induction by sunitinib and improves anti-tumor activity against gastric cancer in vivo. Cancer Lett 2010; 296: 249-256.

47. Zhao H, Leppert JT, Peehl DM. A protective role for androgen receptor in clear cell renal cell carcinoma based on mining TCGA data. PloS ONE 2016; 11: e0146505.

48. Chen X, Guo X, Zhang H, Xiang Y, Chen J, Yin Y et al. Role of miR-143 targeting KRAS in colorectal tumorigenesis. Oncogene 2009; 28: 1385-1392.

49. Yoshino H, Enokida H, Itesako T, Kojima S, Kinoshita T, Tatarano S et al. Tumor-suppressive microRNA-143/145 cluster targets hexokinase-2 in renal cell carcinoma. Cancer Sci 2013; 104: $1567-1574$.

50. Zhang H, Pu J, Qi T, Qi M, Yang C, Li S et al. MicroRNA-145 inhibits the growth, invasion, metastasis and angiogenesis of neuroblastoma cells through targeting hypoxia-inducible factor 2 alpha. Oncogene 2014; 33: 387-397.

51. Cai J, Guan H, Fang L, Yang Y, Zhu X, Yuan J et al. MicroRNA-374a activates Wnt/betacatenin signaling to promote breast cancer metastasis. J Clin Invest 2013; 123: 566-579.

52. Ma Y, Yang Y, Wang F, Moyer MP, Wei Q, Zhang P et al. Long non-coding RNA CCAL regulates colorectal cancer progression by activating Wnt/beta-catenin signalling pathway via suppression of activator protein 2alpha. Gut 2015; 65: 1494-1504.

53. Zhang PP, Wang XL, Zhao W, Qi B, Yang Q, Wan HY et al. DNA methylation-mediated repression of miR-941 enhances lysine (K)-specific demethylase $6 B$ expression in hepatoma cells. J Biol Chem 2014; 289: 24724-24735. 